\title{
Mammography Segmentation with Maximum Likelihood Active Contours
}

\author{
Peyman Rahmati ${ }^{1}$, Andy Adler ${ }^{1}$, and Ghassan Hamarneh ${ }^{2}$ \\ ${ }^{1}$ Dept. of System and computer Engineering, Carleton University, ON, Canada \\ ${ }^{2}$ School of Computing Science, Simon Fraser University, BC, Canada \\ \{prahmati, adler\}@sce.carleton.ca, hamarneh@sfu.ca
}

\begin{abstract}
We present a computer-aided approach to segmenting suspicious lesions in digital mammograms, based on a novel maximum likelihood active contour model using level sets (MLACMLS). The algorithm estimates the segmentation contour that best separates the lesion from the background using the Gamma distribution to model the intensity of both regions (foreground and background). The Gamma distribution parameters are estimated by the algorithm. We evaluate the performance of MLACMLS on real mammographic images. Our results are compared to those of two leading related methods: The adaptive level set-based segmentation method (ALSSM) and the spiculation segmentation using level sets (SSLS) approach, and show higher segmentation accuracy (MLACMLS: $86.85 \%$ vs. ALSSM: $74.32 \%$ and SSLS: 57.11\%). Moreover, our results are qualitatively compared with those of the Active Contour Without Edge (ACWOE) and show a better performance. Further, the suitability of using ML as the objective function as opposed to the KL divergence and to the energy functional of the ACWOE is also demonstrated. Our algorithm is also shown to be robust to the selection of a required single seed point.
\end{abstract}

Index Terms-Active contour models, computer-aided diagnosis, level sets, maximum likelihood, Gamma distribution, mammography

\section{Introduction}

Mammography has a demonstrated potential for increasing survival rates through early detection of intangible tumors and small lesions (Rangayyan, 2005). Digital mammography uses X-rays to project structures in the 3D female breast onto a 2D image (Rangayyan, 2005). Breast cancer is the leading type of cancer in women and the second most fatal (American Cancer Society, 2006). Tumors appear as 
medium-gray to white areas on digital mammograms (Egan, 1988) and their shapes are described by standardized keywords (The Mosby Medical Encyclopedia, 1992) grouped as oval, irregular, lobulated, or round, whereas their margins are expressed as circumscribed, obscured, ill-defined or spiculated. Spiculated lesions are most often associated with cancerous pathologies (Demirkazık et al., 2003), and the presence of irregularly-shaped masses and spicules increases the probability of malignancy (Jeske et al., 2000). In addition to the variable tumor shape and appearance, the inherently noisy nature of digital mammograms, low contrast of suspicious areas, and ill-defined mass border make lesion segmentation an important and challenging problem.

The current standard of breast cancer detection using mammogram is "single reading of mammograms" in the United States, whereas in many European countries "the double reading" is the standard (Gilbert et al., 2008). A current study in 2008 shows that the breast cancer detection rate using single reading with a mammography Computer Aided Detection (CAD) system could be similar to that of the two readers without computer assistance (Gilbert et al., 2008). In this study 31,057 women undergoing routine screening mammography at three centers in England were randomly assigned to double reading, single reading with CAD, or both (Gilbert et al., 2008). The cancer detection rate for the two standards was measured to be equivalent. The double reading detected 199 of 227 cancers $(87.7 \%)$, while the single reading with CAD detected 198 of 227 cancers (87.2\%) (Gilbert et al., 2008). The single reading with $\mathrm{CAD}$ can be concluded to be an alternative to the double reading (Gilbert et al., 2008). Hence, the single reading with $\mathrm{CAD}$ decreases the image review process time while retaining the same accuracy as the double reading.

Although mammography CAD system involves a preprocessing stage, a segmentation stage, and a classification stage, the success of clinical adoption of CAD depends mainly on the accuracy of the mammography segmentation algorithm (Jay et al., 2004). Nevertheless, there is no specific value of segmentation accuracy that guarantees the success of the overall CAD system. One study considers three different levels of segmentation accuracy for mammogram: near perfect, acceptable, and unacceptable 
(Jay et al., 2004). The unacceptable segmentation leads to a false positive decision (Jay et al., 2004). According to our results, the proposed segmentation method provides acceptable level of segmentation accuracy with an overall accuracy of $86.85 \%$, offering a highly accurate segmentation stage for a CAD system. The developed segmentation method can be applied for treatment planning, disease monitoring, or clinical studies. However, our motivation has been for disease monitoring.

Several approaches have been reported for mammography segmentation, including statistical thresholding (Catarious et al., 2004), texture analysis (Sahiner et al., 1998, 2001), and level sets (Sethian, 2002). Catarious et al. (2004) proposed the Catarious Segmentation Method (CSM) which is an iterative thresholding algorithm that uses Fisher's Linear Discriminant Analysis (LDA) to distinguish pixels inside the initial mass area from those outside. Besides the need for training, the main drawback of statistical segmentation is evident in low contrast images, in which the algorithm erroneously classifies background region pixels as part of a lesion, resulting in over-segmentation. Sahiner et al. (1998) proposed a specialized image transformation called Rubber Band Straightening Transform (RBST) for mammographic image analysis. The textural features derived from RBST are based on the location of the mass border, which makes it vulnerable to noisy, ill defined borders. Sethian (2002) used level sets with the fast marching algorithm to segment masses. To control the evolution of the active (or deformable) zero-level set curve, Sethian used a gradient-based stopping term, which produced less robust results because of the noisy nature of mammograms with ill-defined or obscured borders. Sethian's approach is an example of active contour models (ACM), a class of techniques that have gained considerable attention in the medical image analysis community, since the seminal 1988 work of Kass, Witkin, and Terzopoulos, because of their ability to integrate image pixel data into smooth and connected boundaries. ACMs are classified into two main groups: parametric, e.g. (Kass et al., 1998; Yezzi et al., 1997), and implicit, or level set based active contours (Paragios, 2000; Sethian, 2002; Osher and Paragios, 2003). Although the previous level set approaches were applied to many applications, only a few were applied to the segmentation of mammography (Sethian, 2000; Ball and Bruce, 2007a, 2007b), and these approaches 
did not model the detailed statistical characteristics of mammogram images. Robust mammography segmentation methods would benefit from the topological adaptability and mathematical rigor of level sets based ACM as long as they encode properties specific to the mammographic images. The energy function defining an ACM typically includes cost terms reflecting visual cues which define the target object to be segmented. These terms can be divided into regional terms (e.g. expected pixel intensity), boundary (high intensity gradient magnitude along the object boundary), and shape terms (statistical model of shape variability). The type of information included in the energy function determines the effectiveness and the range of application of the ACM. Leventon et al. (2000) and Rousson and Paragios (2002) performed Principal Component Analysis on signed distance functions to augment levels set based active contours with shape priors. However, lesions do not have a predefined shape hence incorporating shape prior is ineffective. Even more signed distance functions are not closed under linear combinations rendering statistical shape modeling difficult (Changizi and Hamarneh, 2010; Andrews et al., 2011).

In this paper, we propose a novel level set active contour model designed for the delineation of lesions in digital mammograms. The algorithm is based on the maximum likelihood (ML) segmentation model but extended to accommodate a mammography-specific intensity distribution. The proposed level sets (LS) based MLACM (hereinafter referred to as MLACMLS) retains the advantages of the regionbased active contour without edges of Chan-Vese (2001) for dealing with noisy images with weak boundaries and simultaneously integrates our knowledge of the statistical distribution of mammogram gray levels to adapt the region based active contour of Chan-Vese for the digital mammogram. The proposed segmentation algorithm outperforms qualitatively and quantitatively its preceding level set active contour based counterparts as well as a threshold based method (CSM), as a non-active contour based method which has previously been deemed as a successful mammography segmentation method. The main difference of the proposed segmentation method comparing with the previous works (Refregier et al., 1997; Horritt, 1999; Nascimento et al., 2005; Ayed et al., 2005) is that the MLACMLS estimates and updates the shape factor $(K)$ of the Gamma distribution in every iteration of the level set evolution, 
while in the previous works, the shape factor is always a pre-determined constant value for the foreground and the background, see Appendix A. By calculating the shape factor as in the proposed method, the segmentation algorithm is less sensitive to different parameter settings of the imaging system that, in turn, result in Gamma distributions with different parameters. An example of the histogram of images taken using different imaging systems is shown in Fig. 3(b) and Fig. 5(b). The shape of the histogram profile varies for different imaging systems; a property that should be taken into account in the segmentation algorithm. The proposed segmentation algorithm updates the shape factor of the Gamma distribution in every iteration, and is therefore capable of fitting to different histogram profiles, a trait absent from the previous works. Also, Ayed et al. consider the scale factor of the Gamma distribution for the foreground/ the background as the average of the intensities inside/outside the region under evolution. However, such simple averaging approach for updating the scale factor, which is akin to how the mean intensity paramters are updated in the seminal 2001 work of Chan and Vese, is not capable of handling images with heterogeneous intensity patterns as visible in mammogram. Moreover, the two component Gamma distribution is indeed a good fit for native mammography images; however we pair the 2-parameter Gamma-distribution based level set segmentation method (MLACMLS) with a preprocessing filter, fuzzy contrast-limited adaptive histogram equalization (FCLAHE), detailed in section 2, which results in improved accuracy compared to when the FCLAHE is not used.

Several papers have incorporated the image intensity probability density functions (PDFs) into active contour models for image segmentation (Rathi et al., 2006; Georgiou et al., 2007; Michailovich et al., 2007; Sandhu et al., 2008). These papers typically seek to segment image regions by finding an optimum point of a PDF similarity metric, see Georgiou et al. (2007) for an overview. For example, Freedman and Zhang (2004) compare maximizing the Bhattacharyya (BH) measure or minimizing the Kullback-Leibler (KL) divergence for tracking purposes; and Rathi et al. (2006) minimize the BH measure to separate regions of different distributions. Recent works have shown that the choice of 
similarity metric has a significant effect on segmentation performance; for example Michailovich et al. (2007) show the BH measures outperforms the KL divergence, and Sandhu et al. (2008) show a newly developed metric outperforms both BH and KL metrics.

The most similar papers to our work, which focus on spiculation segmentation using level sets, are by Ball and Bruce. They suggested two algorithms, the first is for periphery segmentation of a mass and is named adaptive level set-based segmentation method (ALSSM) (Ball and Bruce, 2007a), and the second is a method for spiculation segmentation using level sets (SSLS) (Ball and Bruce, 2007b). The SSLS is a two-stage algorithm; it first uses the Generalized Dixon and Taylor Line Operator (GDTLO), an extended version of the DTLO, to detect the spicules, and then merges the detected spicules with the periphery segmentation of the mass, obtained from ALSSM. The segmentation method proposed in this paper (MLACMLS) analyzes tumors of any type (spiculated or otherwise) under a single, variational energy-minimizing framework. Results show improved segmentation at lower computational cost and without the need for post-processing such as merging. Our approach has similarity to previous active contour based models. Chesnaud et al. (1999) presented a statistical region based active contour adapted to different noise models using ML criterion. They proposed a general model for different statistical laws. To devise a general formula for all images, some of the considered statistical distributions (such as Gamma distribution) were simplified to one-parameter statistical distributions (i.e. unity shape factor). Such simplified distributions lead to less precise models of the image intensity in mammograms. In our approach, we adopt the Gamma distribution for the foreground and background without any simplification to model mammograms and perform the segmentation (section 2.3).

In this work, we focus more on spicules segmentation for two reasons: They contain the most significant features for diagnosing a mass as malignant, and the segmentation of spicules continues to defy an automatic and accurate solution. We test our method on the Digital Database for Screening Mammography (DDSM) (Suckling et al., 1994), which contains low contrast mammography images, and acts as a suitable benchmark for evaluating mammography segmentation algorithms. 
Prior to applying MLACMLS to segmenting a suspicious mass in low-contrast mammography images (such as the images from the DDSM database), we process the images with an enhanced version of the contrast-limited adaptive histogram equalization (CLAHE) (Pisano et al., 2000), which we referred to as the fuzzy CLAHE (FCLAHE) (Rahmati et al., 2010). FCLAHE enhances the original mammographic image while minimizing the effect of changing the statistical distribution of its intensity, for more details see section 2 .

The remainder of the paper is organized as follows: In the next section, we review the FCLAHE as the preprocessing filter for mammogram. In section 3, we first outline the motivation behind choosing the Gamma distribution to model the characteristics of the gray levels of digital mammograms (section 3.1). In section 3.2, we review the ML segmentation method. We present the mathematical details of MLACMLS in section 3.3. In section 4, we present extensive experimental results including: evaluating the robustness of MLACMLS with respect to seed point selection and its single tunable parameter; the superiority of choosing the Gamma distribution for modeling mammograms over the Rayleigh distribution and the Poisson distribution (a pre-assumed distribution to be adequate for modeling the intensity of mammogram); the favorable performance of MLACMLS over SSLS and ALSSM; and the suitability of adopting the ML as the objective function for the active contour as opposed to maximizing the KL divergence method (Georgiou et al., 2007). We finally present discussion and conclusions in section 5 .

\section{Preprocessing Filter (FClahe)}

In this section, we review the applied preprocessing filter (the FCLAHE) for mammogram proposed in our previous work (Rahmati et al., 2010). As per the conclusions of Pisano et al. on the efficacy of the CLAHE (Pisano et al., 2000), the original mammographic images are first processed using the CLAHE to eliminate noise and then the result is processed using a nonlinear filter, which incorporates the advantages of a nonlinear fuzzy function. We integrate these steps into the FCLAHE algorithm as 
follows. We use the fuzzy function proposed by Guliato et al. (2003a) within a new form to provide a non-linear adjustment to the image based on its gray level statistics. The fuzzy function assigns a membership value between 0 and 1 to the pixels based on the difference between their gray levels and a seed point, located at the mass core. The fuzzy function is defined as (Guliato et al., 2003a):

$$
F(p)=\frac{1}{1+\beta|d|}
$$

where $p$ is the intensity of the pixel being processed, $d$ is the intensity difference between $p$ and a seed point, and $\beta$ controls the opening of the function. The larger the difference, the lower the membership; and as $\beta$ increases, the opening of the $F(p)$ decreases. Fig. 1 visualizes the behavior of this function.

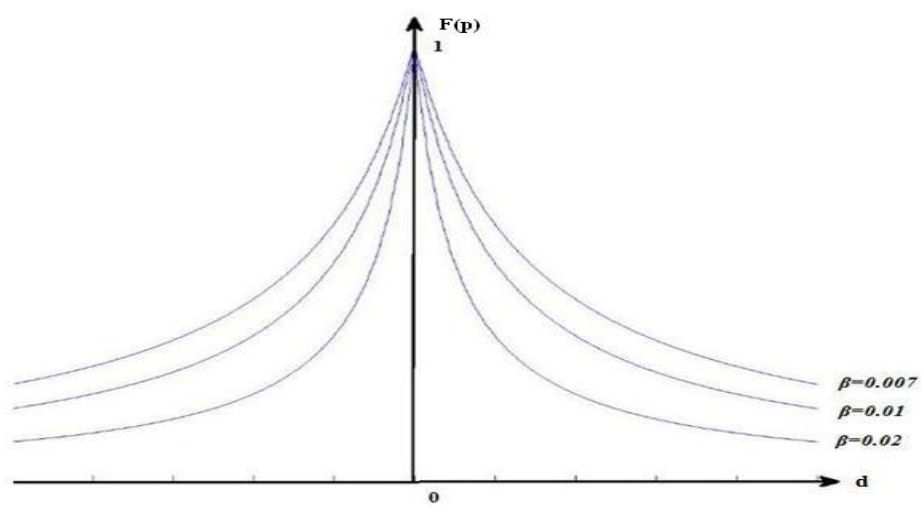

Fig. 1. Fuzzy function behavior of (1) for various values of $\beta$.

The non-linear filter called fuzzy contrast-limited adaptive histogram equalization (FCLAHE) transforms the gray level intensities as follows:

$I_{\text {new }}(x, y)=$

$A_{r}\left(I_{\text {orig }}(X, Y)\right)+\left(I_{\text {orig }}(x, y)-A_{r}\left(I_{\text {orig }}(X, Y)\right)\right) F\left(\left|I_{\text {orig }}(x, y)-A_{r}\left(I_{\text {orig }}(X, Y)\right)\right|\right)$,

where $I_{\text {new }}(x, y)$ is the new intensity, $X=x-x_{\text {seed }}, Y=y-y_{\text {seed }}$. The seed point is selected, by an expert user, e.g. a radiologist, in the center of the mass core, the brightest region in mammograms. $A_{r}\left(I_{\text {orig }}(X, Y)\right)$ denotes the average of the gray levels of the pixels within a radial 
distance $r=\sqrt{(X)^{2}+(Y)^{2}}$ away from a seed point. $I_{\text {orig }}(x, y)$ is the original intensity of a pixel located at the coordinate $(x, y) . F(p)$ is the fuzzy function defined in (1). In (2), it is assumed that the intensities of the pixels at a distance ( $r$ ) away from the seed point are similar unless there are inhomogeneities in the background. Wherever there is a region of high contrast in the background, this region will result in higher values for $I_{\text {orig }}(x, y)$ and hence it will be more different than $A_{r}$, i.e. $\left(\mid I_{\text {orig }}(x, y)-\right.$ $\left.A_{r}\left(I_{\text {orig }}(X, Y)\right) \mid\right)=\Delta I_{r}(x, y)$ will give a higher value. According to $(1), F\left(\Delta I_{r}(x, y)\right)$, will therefore yield a lower value. This, in turn, will attenuate the second term in the right hand side of (2), and $I_{\text {new }}(x, y)$ will be assigned $A_{r}$. If there are no inhomogenieties in the background, then $A_{r}$ will be close to $I_{\text {orig }}(x, y)$, i.e. $\Delta I_{r}(x, y) \approx 0$, and $F(0)$ will yield unity, which in turn assigns $I_{\text {new }}(x, y)$ to $I_{\text {orig }}(x, y)$, (i.e. the intensity of the pixel does not change). An example of how FCLAHE filter works on a real image is demonstrated in Fig. 2.

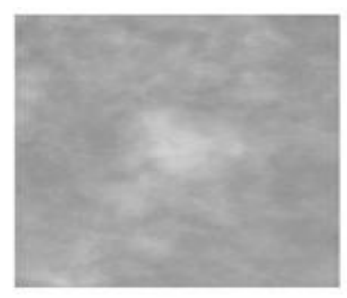

(a)

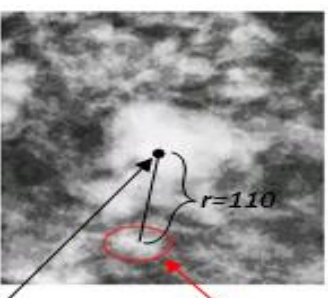

(b)

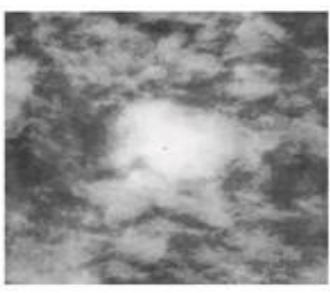

(c)

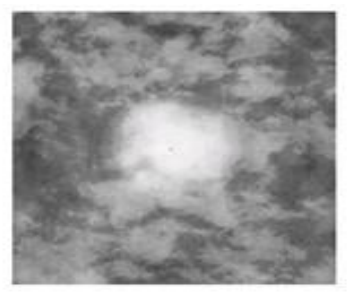

(d)

(e)

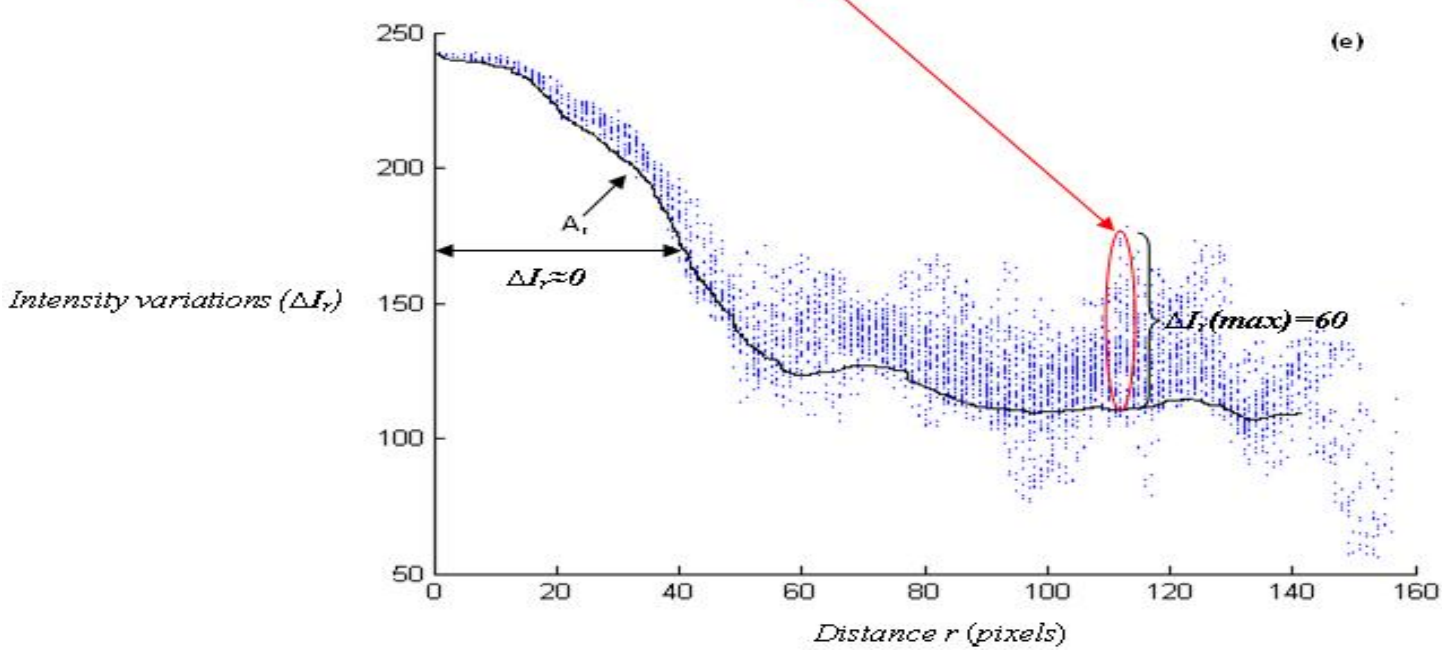

Fig. 2. Example of applying the FCLAHE algorithm on a mammographic image. (a) Original 
mammographic image from DDSM. (b) The image in (a) enhanced using CLAHE has a good contrast but some inhomogeneities (one of them is marked by the red oval) in the background may be mistaken for a real mass. (c) The image (a) enhanced using our proposed FCLAHE with $\beta=0.01$ has an acceptable contrast for the main mass only while significantly reducing the contrast in the background. (d) FCLAHE image with $\beta=0.02$ possesses the same characteristics as in (c). (e) The intensity variations of pixels located a radial distance $r$ from the seed point. In particular, the effect of the inhomogeneous background of the tumor at a distance of 110 is highlighted in (e) by the red oval. The highest intensity variation of 60 occurring at distance 110 is due to the inhomogeneous region marked by the red oval in (b). The solid line in black in (e) represents the average intensity value of pixels placed a distance $r$ away from the seed point.

In fact, equation (2) removes inhomogeneities; which may be mistaken for a real lesion in the subsequent segmentation algorithm, and simultaneously preserves the intensity of the mass core, e.g. $r<40$ in Fig. 2(e). The advantage of the FCLAHE filter is its ability to eliminate noise while preserving the intensity characteristics of the mammographic image (Figure 2(c), (d)). This feature of FCLAHE is crucial for the subsequent segmentation approach which relies on analyzing the statistical distribution of the intensities in the mammogram.

\section{Methods}

\section{1. Statistical Distribution of Image Intensity in Mammograms}

The proposed approach is motivated by the need to accurately model the statistical properties of a mammography image. There is a strong dependence between the segmentation accuracy and the PDF of the noise present in the image (Martin et al., 2004). In this work ${ }^{1}$, we improve the mammography segmentation accuracy by adapting the Gamma distribution, offering two tunable parameters. The two parameters demand more computations to evolve the MLACMLS (Appendix A and B). Nevertheless, we adopt the 2-parameter Gamma distribution for modeling the PDF of mammograms because, first, tumors appear as medium-gray to white areas on digital mammograms (Egan, 1988) with a relatively long tail in their histogram (Figure 3) and, second, because there is a wide range of databases for mammograms

\footnotetext{
${ }^{1}$ Preliminary version of using MLACMLS for mass segmentation appeared in our earlier work (Rahmati and Ayatollahi, 2009), in which Poisson distribution with a single tunable parameter was used.
} 
acquired using different devices and settings, so a two-parameter distribution allows more flexibility to adapt the PDF to this variability compared to a one-parameter model, e.g. adapting to distributions that can vary from the one in Fig. 3(b) with a long tail to the one in Fig. 5 (b) with a very short tail. A oneparameter distribution, such as the Rayleigh distribution or the Poisson distribution, is sensitive to the intensity variations of mammogram as they model a limited range of intensity variations, and finally, our empirical results (section 4.4) show that the Gamma distribution provides a better fit to mammography data compared to the Poisson distribution that has a single parameter for controlling the tail of the distribution and to the 1-parameter Rayleigh distribution.

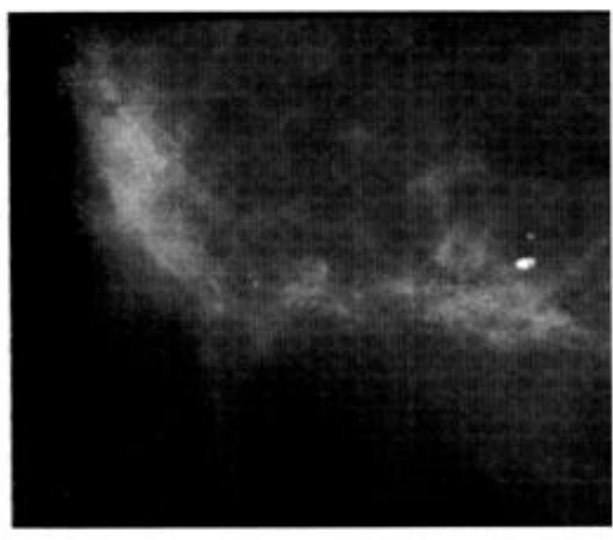

(a)

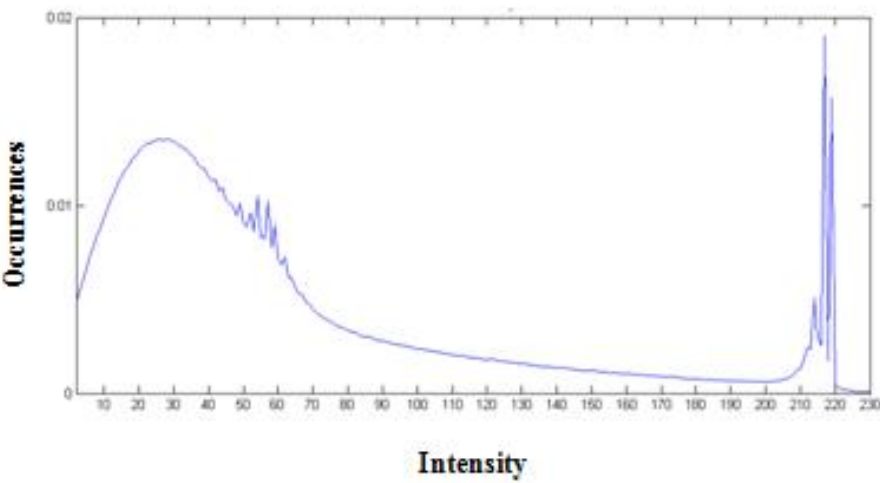

(b)

Fig. 3. Example histogram of a typical mammographic image. (a) A spiculated malignant tumor (reproduced with permission from Guliato et al. (2003)). (b) The histogram of (a), which depicts a long tail connecting the two peaks describing the main tumor (the narrow band peak at intensity 215) and its background (the wide band peak around intensity 25). Note that the histogram is without applying any filtering.

\section{2. ML Segmentation}

We use the ML segmentation in a new level set based ML form specialized for mammographic images (section 3. 3). The ML segmentation considers an image $I$ as a real positive function defined on a rectangular domain $\Omega \subset R^{2}$ (Sarti et al., 2005) with PDF $p(I)$. An initial closed curve $C$ divides the 
image $I$ into an inside region $\Omega_{i}$ and an outside region $\Omega_{e} . P_{i}=\prod_{\left.\Omega_{\mathrm{i}} \mathrm{C}\right)} p(I(x, y))$ is the probability of the random field inside the curve and $P_{e}=\prod_{\Omega_{\mathrm{e}}(\mathrm{C})} p(I(x, y))$ is the probability outside the curve (Sarti et al., 2005). Assuming independence between pixel intensities, the likelihood function is given by the product of the inner and the outer probability (Azzalini, 1996), i.e. $P[I \mid C]=P_{i} P_{e}$. Then the curve $C$ that maximizes this likelihood function is considered the solution to the segmentation problem. In the continuous formulation, the log likelihood function $l$ can be written as (Sarti et al., 2005):

$l(I, C)=\log P_{i}+\log P_{e}=\int_{\Omega_{i(C)}} \log (p(I(x, y))) d x d y+\int_{\Omega_{e(C)}} \log (p(I(x, y))) d x d y$

\section{3. ML active contour model using level sets (MLACMLS)}

\subsubsection{The energy functional}

Following the justification of section 3.1, we substitute the Gamma distribution into (3) with different parameters for inside and outside the contour. The Gamma distribution is defined as:

$$
P(I(x, y))_{G a m m a}=I(x, y)^{k-1} \frac{e^{\frac{-I(x, y)}{\theta}}}{\theta^{k} \Gamma(k)} \quad, I(x, y)>0, \quad \theta, k>0,
$$

where $k$ is the shape factor, $\theta$ is the scale factor, $\Gamma(k)$ is the Gamma function and $I(x, y)$ is the image intensity. The corresponding log likelihood is given by (Appendix A):

$$
l\left(I, C, \theta_{i}, \theta_{e}, k_{i}, k_{e}\right)=-A_{i} \mathrm{~L}\left(S_{i}\right)-A_{e} \mathrm{~L}\left(S_{e}\right),
$$

where $L(x)=\frac{1}{2 x}(\log (2 x)+1)+\log \left(\Gamma\left(\frac{1}{2 x}\right)\right), A_{i}$ and $A_{e}$ are the number of pixels inside and outside of the curve $C$, respectively, and $S_{i}$ and $S_{e}$ are given by:

$$
\begin{aligned}
& S_{i}=\log \left(\frac{\int_{\Omega_{i}} I(x, y) d x d y}{A_{i}}\right)-\frac{\int_{\Omega_{i}} \log (I(x, y)) d x d y}{A_{i}}, \\
& S_{e}=\log \left(\frac{\int_{\Omega_{e}} I(x, y) d x d y}{A_{e}}\right)-\frac{\int_{\Omega_{e}} \log (I(x, y)) d x d y}{A_{e}} .
\end{aligned}
$$


The estimated parameters of the PDF have been derived in Appendix A and are summarized in equations A5, A6, A15, and A16. The PDF parameter estimation procedure is done after every iteration of the algorithm and the new parameters replace the old ones in the next iteration.

A regularizer term, $R(C)=$ length $(C)$, to smooth the shape of the curve is incorporated into (5) as described by Chan and Vese (2001). The energy functional is now rewritten as:

$$
F(C \mid I)=\mu R(C)-A_{i} \mathrm{~L}\left(S_{i}\right)-A_{e} \mathrm{~L}\left(S_{e}\right)
$$

\subsubsection{Level set formulation}

Prior to minimizing the energy functional by deriving the associated Euler-Lagrange equation, we first replace $C$ in (8) with a level set function $\Phi: \Omega \rightarrow R$, with the inside of the curve now captured by $\Phi(x, y)>0$, outside the curve by $\Phi(x, y)<0$, and the curve $C$ itself is given by $\{(x, y) ; \Phi(x, y)=0\}$. With $H(\Phi)$ denoting the Heaviside function, the energy functional can be rewritten as:

$F(\Phi \mid I)=\mu \int_{\Omega}|\nabla H(\Phi)| d x d y-\left[A_{i} \mathrm{~L}\left(S_{i}\right)+A_{e} \mathrm{~L}\left(S_{e}\right)\right]$

in which:

$S_{i}=\log \left(\frac{\int_{\Omega} I(x, y) H(\Phi) d x d y}{\int_{\Omega} H(\Phi) d x d y}\right)-\frac{\int_{\Omega} \log (I(x, y)) H(\Phi) d x d y}{\int_{\Omega} H(\Phi) d x d y}$,

$S_{e}=\log \left(\frac{\int_{\Omega} I(x, y)(1-H(\Phi)) d x d y}{\int_{\Omega}(1-H(\Phi)) d x d y}\right)-\frac{\int_{\Omega} \log (I(x, y))(1-H(\Phi)) d x d y}{\int_{\Omega}(1-H(\Phi)) d x d y}$.

After computing the first variation of (9) (Appendix B), the derived Euler-Lagrange equation is expressed as:

$\partial_{t} \Phi=\delta(\Phi)\left[\mu \operatorname{div}\left(\frac{\nabla \Phi}{|\nabla \Phi|}\right)+\frac{\left(1+\log \left(2 S_{i}\right)\right)}{2 S_{i}}-\frac{C_{i}}{2 S_{i}^{2}} \log \left(2 S_{i}\right) c_{i 1}+\log \left(\Gamma\left(\frac{1}{2 S_{i}}\right)\right)-\Psi\left(\frac{1}{2 S_{i}}\right)\left(\frac{C_{i}}{2 S_{i}{ }^{2}}\right) c_{i 1}-\right.$

$\left.\frac{\left(1+\log \left(2 S_{e}\right)\right)}{2 S_{e}}-\frac{C_{e}}{2 S_{e}^{2}} \log \left(2 S_{e}\right) c_{e 1}-\log \left(\Gamma\left(\frac{1}{2 S_{e}}\right)\right)-\Psi\left(\frac{1}{2 S_{e}}\right)\left(\frac{C_{e}}{2 S_{e}^{2}}\right) c_{e 1}\right]$.

where $\delta($.$) is the dirac delta, \Psi$ is the Digamma function, $C_{i}$ and $C_{e}$ are two auxiliary vectors introduced in the appendix B, $c_{i 1}=\int_{\Omega} \mathrm{H}_{\varepsilon}(\Phi) \mathrm{dxdy}$, and $c_{e 1}=\int_{\Omega}\left(1-H_{\varepsilon}(\Phi)\right) d x d y$.

Finally, the segmentation update equation is given by: 


$$
\left\{\begin{array}{cc}
\partial_{t} \Phi=\delta(\Phi)\left[\mu \operatorname{div}\left(\frac{\nabla \Phi}{|\nabla \Phi|}\right)+\frac{\left(1+\log \left(2 S_{i}\right)\right)}{2 S_{i}}-\frac{C_{i}}{2 S_{i}^{2}}\right. & \log \left(2 S_{i}\right) c_{i 1}+\log \left(\Gamma\left(\frac{1}{2 S_{i}}\right)\right)- \\
\Psi\left(\frac{1}{2 S_{i}}\right)\left(\frac{C_{i}}{2 S_{i}^{2}}\right) c_{i 1}-\frac{\left(1+\log \left(2 S_{e}\right)\right)}{2 S_{e}}-\frac{C_{e}}{2 S_{e}^{2}} \log \left(2 S_{e}\right) c_{e 1}- \\
\left.\log \left(\Gamma\left(\frac{1}{2 S_{e}}\right)\right)-\Psi\left(\frac{1}{2 S_{e}}\right)\left(\frac{C_{e}}{2 S_{e}^{2}}\right) c_{e 1}\right], & \text { in } \Omega \times(0, \infty), \\
\Phi(x, y, 0)=\Phi_{0}(x, y) & \text { in } \Omega \\
\frac{\delta(\Phi)}{|\nabla \Phi|} \frac{\partial \Phi}{\partial \vec{n}}=0 & \text { on } \Omega
\end{array}\right\}
$$

where $\Phi(x, y, 0)$ denotes an initial function defined by $\Phi(x, y, 0)= \pm d$, where $d$ is the signed distance function (Sethian, 1996), and $\vec{n}$ denotes the exterior normal vector to the boundary $\partial \Omega$. The level set evolution process is terminated when the image domain is segmented in two maximally homogeneous areas, as the inside and the outside of the curve. We have applied the Sussman reinitializing procedure (Sussman et al., 1994) to prevent the level set function from becoming too steep or too smooth. The proposed segmentation algorithm is summarized in table 1.

\section{Table 1}

Summary of the proposed segmentation algorithm.

1. Apply the preprocessing filter on the original image using equation (2).

2. Initialize a contour in the domain of the image.

3. Calculate the signed distance function $\Phi$ of the initial contour.

4. Calculate $\partial_{t} \Phi$ using equation (13).

5. Update $\Phi$ using $\Phi_{\text {new }}=\Phi_{\text {old }}+\partial t . \partial_{t} \Phi$.

6. Repeat steps 4 and 5 until convergence (change in $\Phi$ is less than a threshold). 


\section{Results}

We tested the method on 100 test images including 50 spiculated tumors and 50 other types of masses selected from the DDSM database. The 100 images selected are those deemed by two expert radiologists as containing a hard to segment tumor. To account for inter-rater variability in the ground truth, in addition to the manually drawn contours in DDSM, two expert radiologists provided two additional delineations of the tumors in the mammographic images. For each image, the ground truth delineation was obtained by following the rule that a pixel belongs to the mass when it is included in at least two out of the three delineations drawn by the experts (i.e. a majority vote).

The only manual operator intervention needed in our implementation is the placing of one seed point approximately in the middle of the mass core. Then, a square ROI sized $256 \times 256$ pixels (116.4 $\mathrm{mm}^{2}$ ) centered at the seed point is selected. The ROI must contain the tumor to be segmented, and one tumor should segmented at a time (i.e. one tumor per ROI). If more tumors need to be segmented, then several ROIs (one for each tumor) must be used. The size of the tumors in our data set was not more than $256 \times 256$; however, if the tumor can potentially occupy an area larger than $256 \times 256$, then larger ROI must be used. Prior to segmentation, we filter the images with the FCLAHE filter, which requires the setting of an "openness" parameter $\beta$ (Rahmati et al., 2010). The FCLAHE is applied only for the MLACMLS; however, the other competing methods benefit from applying the noise reduction filter proposed in their respective papers. $\beta$ was optimized on a random training set of 80 images and fixed across the remaining 20 test images. The optimization procedure was repeated 5 times to test on all 100 images (blocked-based cross validation). The optimality criterion was the overlap against the ground truth, measured using the Jaccard similarity coefficient (Jaccard, 1908) defined in (14). Then, $\beta$ was fixed for all our experiments at an average value of 0.012. Similarly, the length parameter $\mu$ in (13) was fixed at 0.0012 for all test images. The value of $\mu$ relates to the size of the tumor under investigation; smaller tumors, such as calcifications, require smaller $\mu$ to be detected, and vice versa. According to our 
experiments, the possible range of values for the shape parameter over our data base is between 40 and 140 and, albeit small, the values of scale factors in A5 and A6 do not approach zero.

The proposed segmentation algorithm was implemented and tested on a Pentium IV (PC), Intel 3.0 GHz, with Windows XP Professional, 3.0 GB RAM, in MATLAB 7.0 (Mathworks, Natwick, USA). The time needed to process one $256 \times 256$ image varied from 10 to 60 seconds depending on the initial contour position and the type of the lesion under investigation. The spiculated masses required longer time (about 1 minute) to analyze because the segmentation needs to be performed for the spicules around the mass core as well. The calculation of the sign distance function, step 3 in table 1, requires an average of 0.0014 seconds. To calculate $\partial_{t} \Phi$, step 4 in table 1 , the following parameters must be updated: Length parameter (the first term in (13)), $S_{i}, S_{e}, C_{i}, C_{e}, c_{i 1}, c_{e 1}$. The average required calculation time for updating the parameters is 0.0177 seconds. With all the parameters updated, the calculation of $\partial_{t} \Phi$ requires, on average, 0.0028 seconds. Our implementation uses optimized matrix operations. In total, each iteration takes about 0.1192 seconds. As shown in figure 5, at most 500 iterations were sufficient for convergence. For further speed improvements, parallelization and hardware acceleration may be explored, but were deemed unnecessary for our purposes.

The proposed segmentation method has been applied to two types of tumors: non-spiculated tumors (Figure 4) and spiculated tumors (Figures 5-7). To evaluate the segmentation accuracy of our proposed method (MLACMLS), we compare it with ALSSM and SSLS, since these are the closest to our work, are the most recent state-of-the-art methods that use level sets for spiculation segmentation with promising results, and both of them have been previously tested using the DDSM. For the competing methods (ALSSM and SSLS), we implemented the algorithm following the details provided in those papers. The implementations were validated by testing them in images and observing similar results to those reported in the original papers. In comparison to SSLS and ALSSM, we note the following advantages that our method offers: we use the same level sets algorithm to segment both spiculated and 
non-spiculated tumors, independent of the type of tumors (Figures 4 and Figure 7); we provide an automatic segmentation algorithm with higher accuracy in tumor delineation, including spicules, demonstrating lower sensitivity to the variations in the algorithm's parameters (Figures 11 and Figure 12); and, finally, we are able to detect the thickness of distinct spicules more precisely (Figure 7).

Qualitative results are shown, in section 4.1, for a subset of the database (to conserve space), while the quantitative analysis is performed over the complete aforementioned dataset of 100 images. Quantitative analysis, in section 4.2, includes evaluating the assumption of Gamma distribution and comparing our results with the delineation drawn by experts. This comparison is evaluated in terms of segmentation accuracy, calculated using an overlap measure (defined in section 4.2), the error in extracted shape features' values, and the correlation coefficient of each single shape feature with the ground truth. Detailed quantitative results are given in Section 4.2; however, we summarize the results here for convenience. MLACMLS achieved the lowest error and highest correlation values. In particular, MLACMLS achieved a segmentation accuracy of $86.85 \%$ (vs. $57.11 \%$ for SSLS and $74.32 \%$ for ALSSM) averaged over the delineation of all tumors from each type in our database, with an overall average error in the obtained shape features' values of 0.0299 (vs. 0.0768 for SSLS and 0.0321 for ALSSM) and average shape features correlation coefficient of 0.8395 (vs. 0.7052 for SSLS and 0.7105 for ALSSM).

To investigate the assumption of Gamma distribution on all the images, first, the similarity between the PDF estimated by the proposed algorithm and the histogram of the mammographic image is evaluated (Figure 5). Then, the Kolmogorov Smirnov test in Georgiou and Chen (1998) is applied to evaluate the similarity between the estimated PDF and the real histogram of the mammogram. The test was performed on all the images with a fixed openness value of 0.012. The hypothesis of Gamma distribution was admitted on 96 out of the 100 images when setting the test significance at 0.05 . The four failed images were of spiculated tumors with ill-defined edges. Changing the openness value to 0.01 for these four images only resulted in admitting the hypothesis test. 
Moreover, the proposed segmentation method has an acceptable robustness for both seed point selection and $\beta$ variations where the overlap decreased by at most $10 \%$ for choosing different seed points and by at most $12 \%$ for $\beta$ values in the range [0.01, 0.02], see section 4.3 . In section 4.4 , we compare the performance of the MLACMLS when adapting three different PDFs (Gamma distribution, Poisson distribution, and Rayleigh distribution), resulting in the best average segmentation accuracy of $86.85 \%$ for the Gamma distribution vs. $81 \%$ for Poisson distribution and unsuccessful segmentation results for Rayleigh distribution. The performance of the proposed log-likelihood function as objective function which evolves the contour is compared to that of $\mathrm{KL}$ divergence metric in section 4.5 , offering the superiority of the proposed objective function.

The proposed segmentation algorithm showed less sensitivity to the initialization: when perturbing the size of the initial contour by $100 \%$ the segmentation results did not change more than $8 \%$, the sensitivity to changing the shape of the initial contour from a box to a circle was less than $4 \%$, and the segmentation accuracy perturbed by less than $10 \%$ when changing the location of the initial contour from being at the boundary of an inclusion to completely inside the inclusion.

\subsection{Qualitative comparison results}

In this section, some typical pathological cases are presented along with the segmentation results obtained with the MLACMLS, SSLS, ALSSM and CSM, as well as the physician-supplied ROI and the ground truth. We present the same study cases in Ball and Bruce (2007a, 2007b). In Fig. 4, we have considered non-spiculated lesions. 

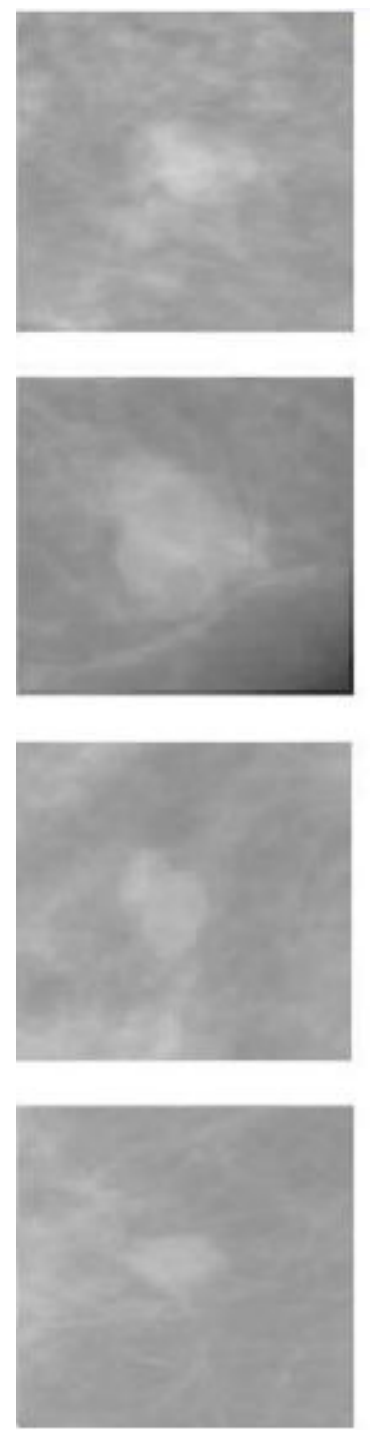

Fig.

4. Qualitative segmentation results on four sample images. (left column) Original mammogram image. (middle column) CLAHE (Pisano et al., 2000) enhanced image with overlays of the DDSM ROI (white line), ALSSM segmentation (black line) and CSM segmentation (black and white dashed line) reproduced with permission from Ball and Bruce (2007a). (right column) Final segmentation image of MLACMLS algorithm performed on the enhanced image with FCLAHE (green line) in comparison with the ground truth (white line). The different rows represent different example cases.

The MLACMLS and ALSSM results are more similar to the ground truth than the CSM when segmenting non-spiculated masses. The CSM result has, except in two cases (Fig. 4 (a) and (c)), some relatively noticeable differences in comparison with the ground truth; it can be seen that the CSM oversegments while the ALSSM tends to slightly under-segment in some cases (Fig. 4(a) and (b)). We note that MLACMLS is a good compromise between these two previously developed methods. Finally, as per 
the ground truth in Fig. 4, the accuracy of the MLACMLS to segment non-spiculated tumors appears higher in comparison with other previously developed algorithms.

Fig. 5 and Fig. 6 depict the iteration process of MLACMLS for spicules, selected from two different datasets (DDSM and Guliato et al., 2003a). The fitness curve and the behavior of log likelihood functions along the evolution are also visualized in Fig. 5. The plateau in the log likelihood curve $l$ is an indication that the curve $C$ has segmented the image into two maximally similar regions as foreground and background. The fitness curve in Fig. 5 (b) represents a good agreement between the estimated PDF of MLACMLS and the real histogram.

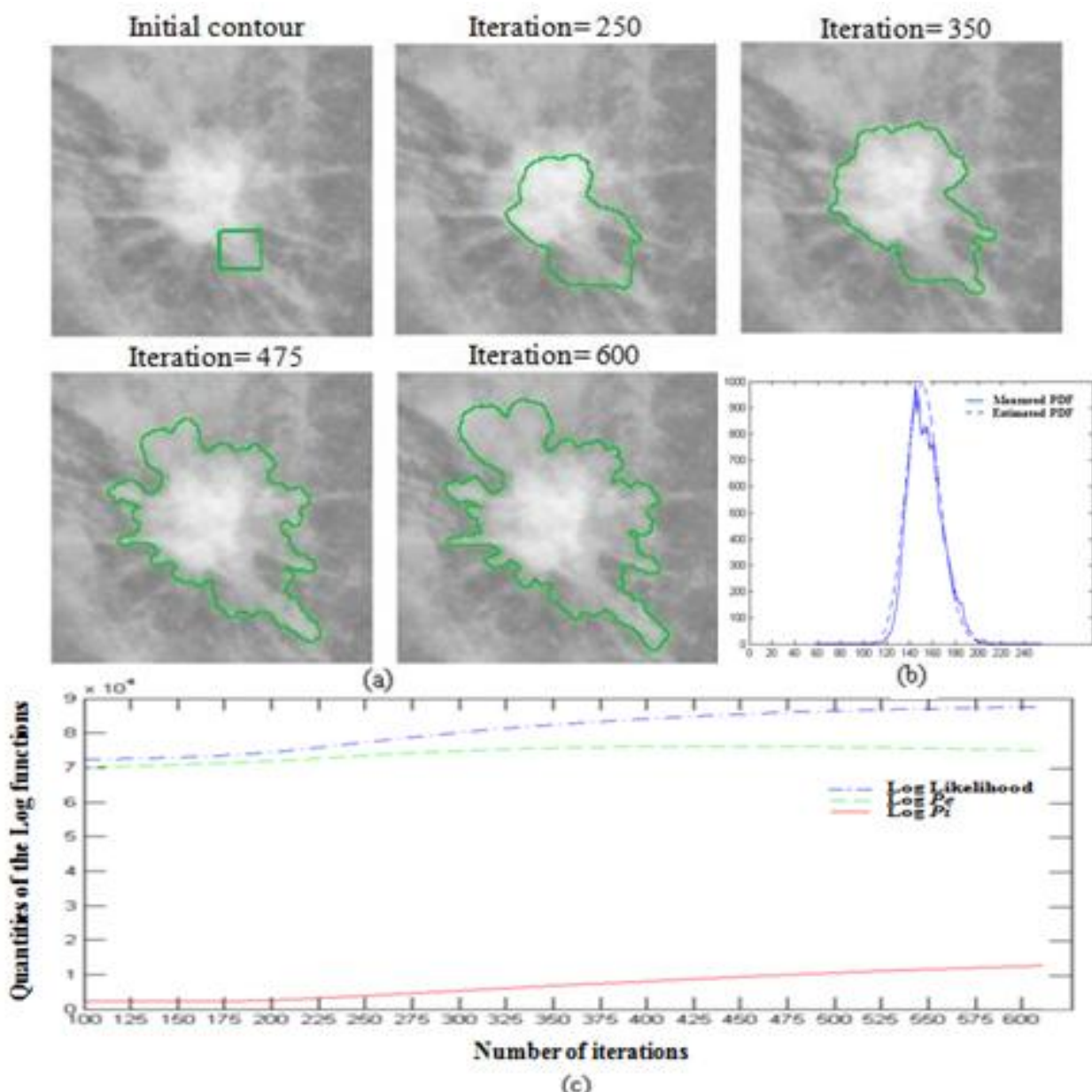

Fig. 5. Segmentation of a spiculated tumor from the DDSM database. (a) Evolution of the initial curve (the first row as well as the first and the middle column of the second row). (b) The estimated Gamma probability density function (dotted) and the measured histogram of the mammogram image background 
(solid) (c) The behavior of the log likelihood functions during the evolution of the curve $C$, including $\log$ $p_{i}$ (solid red line), $\log p_{e}$ (dashed green line), and log likelihood $l$ (point dashed blue line). Note that the original image is already filtered by the FCLAHE.

In Fig. 5, the evolution of the curve $C$ for a hard to segment, spiculated, malignant tumor, reproduced from Guliato et al. (2003a), using MLACMLS is visualized. The final segmentation result of MLACMLS shows an acceptable segmentation result in comparison with the expert delineation reported in Guliato et al. (2003a).

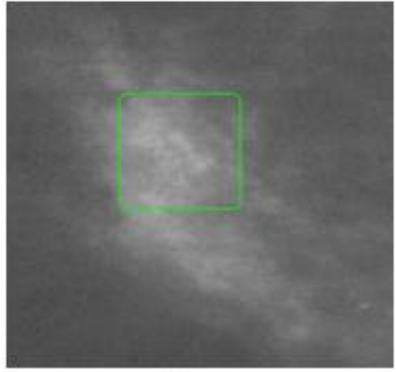

(a)

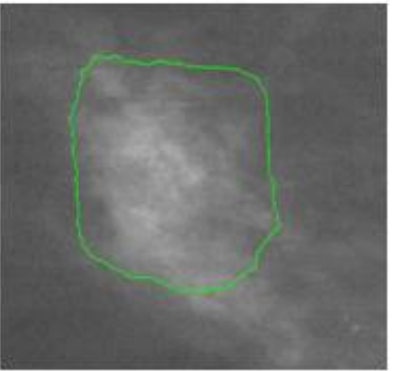

(b)

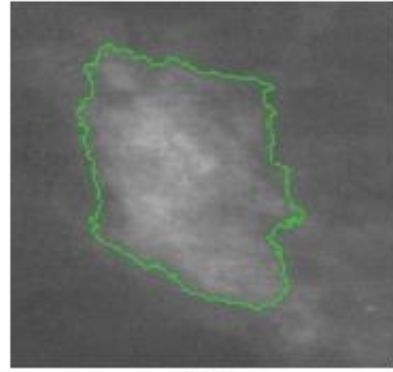

(c)

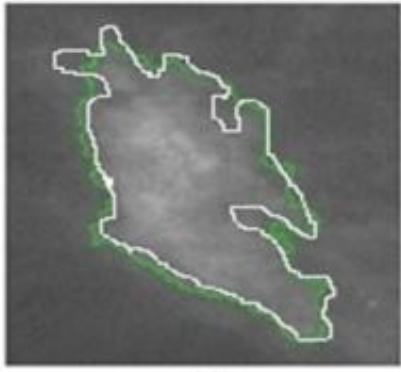

(d)

Fig. 6. Segmentation of a malignant spiculated tumor, reproduced with permission from Guliato et al. (2003a), using the MLACMLS. (a) Initial condition. (b), (c) two steps of the contour evolution. (d) The final delineation in green which has a close agreement with the region delineated by an expert in white. Note that the original image is already filtered by the FCLAHE.

The comparison of the segmentation results of the SSLS and the MLACMLS with the ground truth for some spiculated tumors, achieved from DDSM database is depicted in Fig. 7. As shown, there is a low similarity between the SSLS and the ground truth. The MLACMLS clearly outperforms the SSLS in delineating spiculated tumors in these examples. Also, the thickness of the distinctive spicules is visually detected with more precision than the SSLS. Fig. 6 and 7 show examples of the performance of our proposed algorithm when applied to images captured by different imaging systems. 

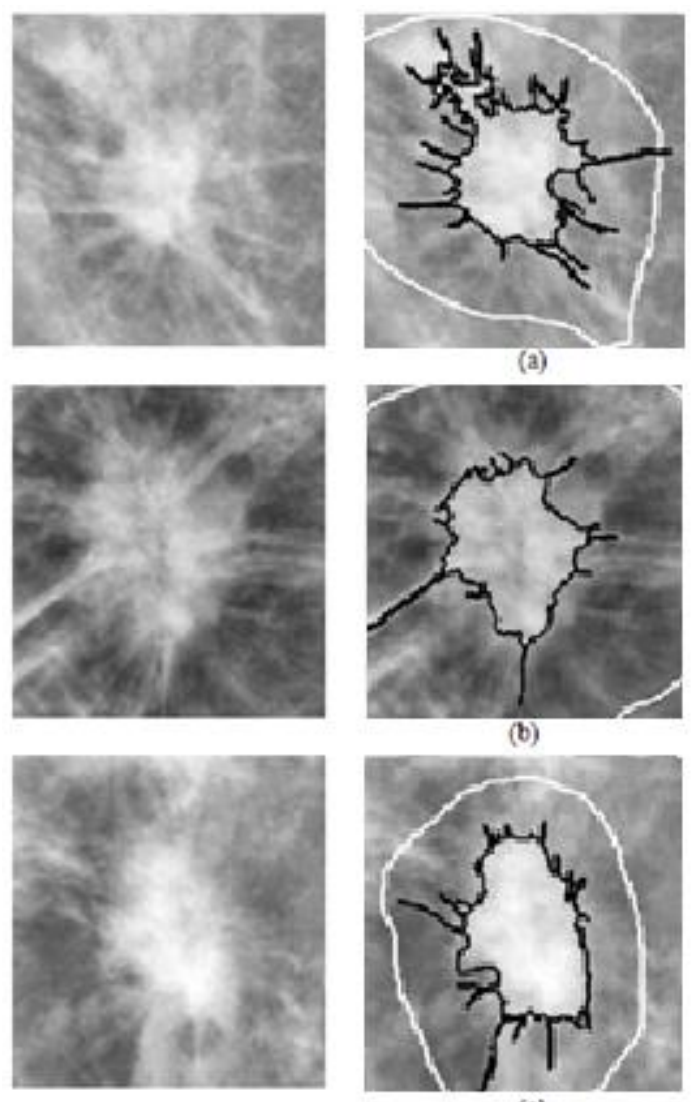

(a)

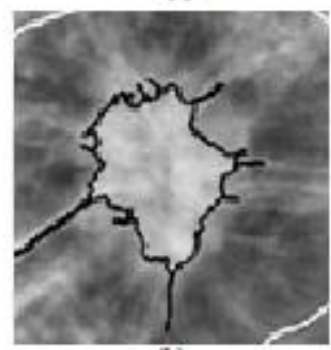

(b)

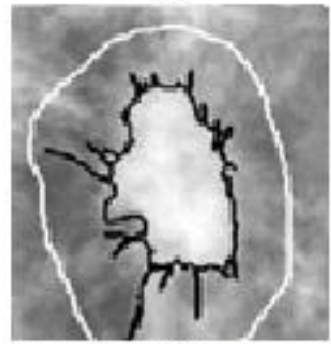

(c)
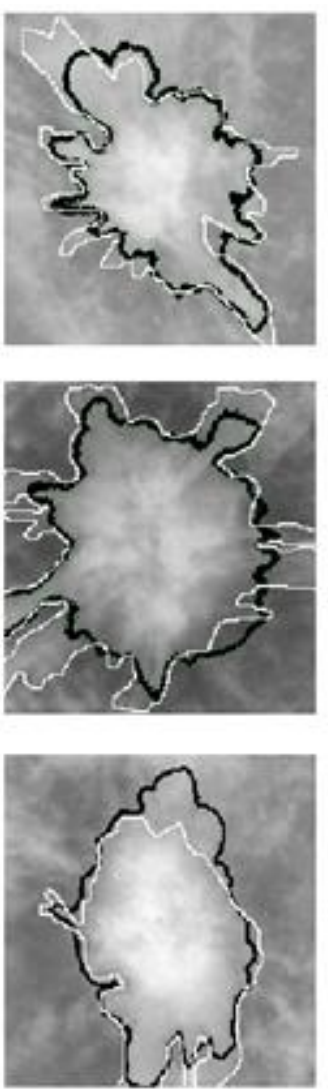

Fig. 7. Examples of the spiculation segmentation. (left column) CLAHE filtered image. (middle column) SSLS segmentation with overlays of the physician-supplied ROI (white line) reproduced with permission from Ball and Bruce (2007b). (right column) MLACMLS delineation (black line) applied to the enhanced image using FCLAHE with the contour of ground truth (white line).

The comparison between the MLACMLS and Active Contour without Edge (ACWOE) of Chan and Vese is shown in Fig. 8. Although the results of both methods for different initialization (small, large, and tiled) and different tumor types (non-spiculated and spiculated) are somewhat similar, the ACWOE fails to consistently minimize the energy function over iterations, producing erroneous false positives in the background for the non-spiculated tumor. The only case that the MLACMLS does not possess a continuously increasing likelihood curve is when using a tiled initialization (right column in both panels of Fig. 8). However, in this case, the likelihood curve is improved after a small number of iterations. The energy function of the ACWOE fails to be minimized when using large initialization (middle column in 
both panels). The MLACMLS qualitatively outperforms the ACWOE in segmenting the non-spiculated tumor and the spiculated tumor when using either small or large initialization (the first and the middle columns in both panels of Fig. 8).

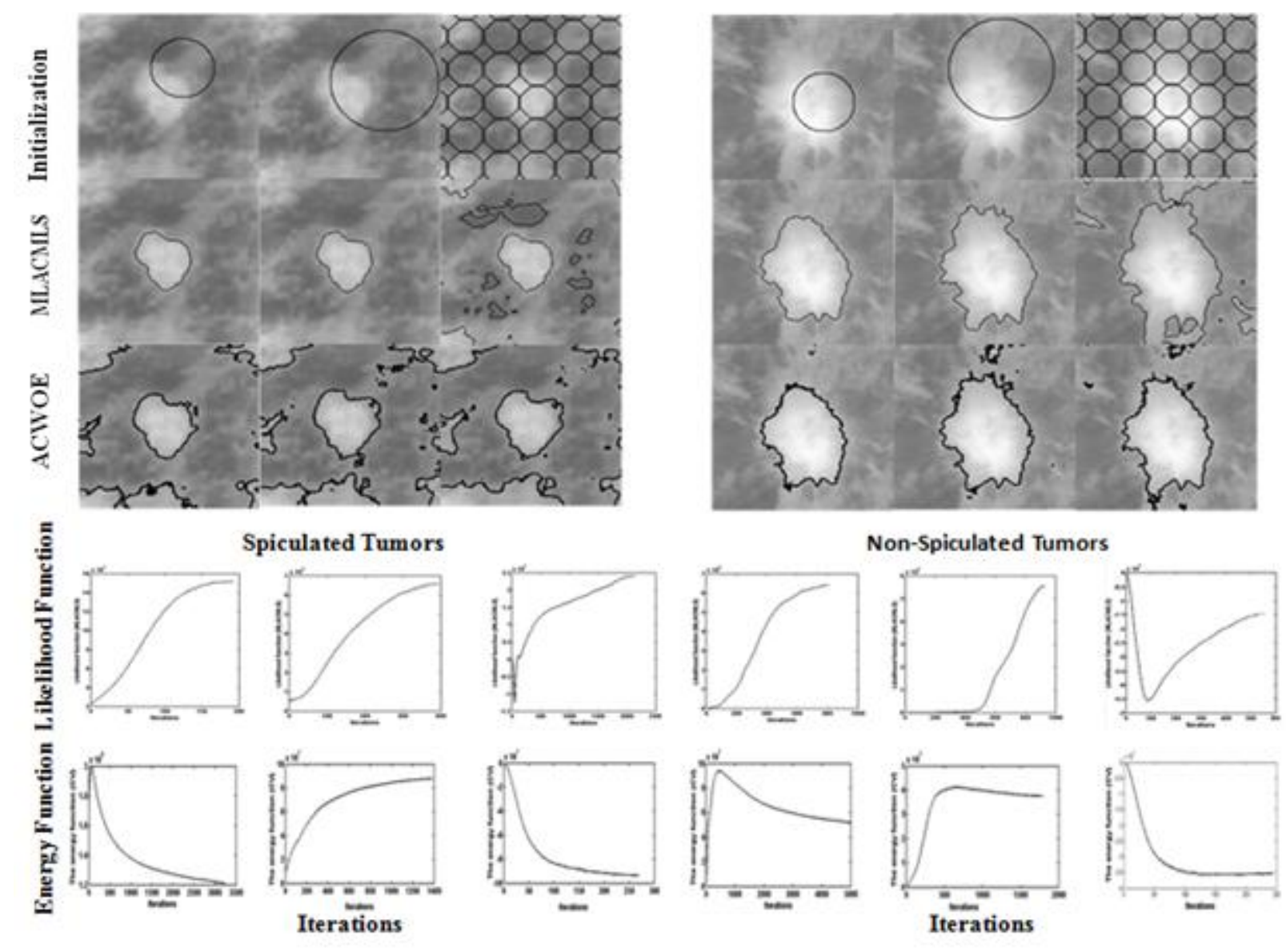

Fig. 8. Comparison between the MLACMLS and the ACWOE sensitivity to initialization. Two types of images: spiculated on the left panel and non-spiculated on the right panel. Three different types of initialization (small, large, tiled) seen in the three columns of each panel. Best parameters chosen for each case (exhaustive search, trial and error, empirically set). First row show initialization overlaid on the original image. Second row shows the result of our MLACMLS and the third row ACWOE. For each case study in each column, the likelihood function of the MLACMLS is represented in the fourth row as well as the energy function of the ACWOE in the fifth row.

\subsection{Quantitative results}

We implemented the competing methods in Ball and Bruce (2007a, 2007b) and compared their performance to the proposed MLACMLS (Tables 2-6). We compare the values of shape descriptors since 
many algorithms rely on shape measures to perform a CAD process. To quantify segmentation accuracy, we use the Jaccard index (Jaccard, 1908) to measure the overlap $(O V)$ between two segmented areas $A$ and $B$ as follows:

$$
O V=\frac{|A \cap B|}{|A \cup B|}
$$

where $A$ is the ground truth delineation, $B$ is the delineated area by the algorithm, and $O V=l$ when $A$ and $B$ match perfectly. The shape features examined are normalized compactness and several moment invariants. In particular, we calculate the normalized compactness as (Shen et al., 1993; Rangayyan et al., 1997):

$$
c f=1-\frac{4 \pi A}{P^{2}}
$$

where $p$ and $A$ are the contour perimeter and enclosed area, respectively. Moreover, we examined a set of shape features invariant to translation, rotation, and scaling and representing the moments of distances to the centroid of the region (Gupta and Srinath, 1987), which are calculated as follows:

$$
m_{p}=\frac{1}{N} \sum_{n=0}^{N-1}[d(n)]^{p},
$$

where $d(n)$ is the Euclidean distances between the centroid of the area and all of the pixels along the contour. The $p^{\text {th }}$ central moment is defined by (Rangayyan et al., 1997):

$$
M_{p}=\frac{1}{N} \sum_{n=0}^{N-1}\left[d(n)-m_{1}\right]^{p} .
$$

where $m_{l}$ is when $p=l$ in (16). Finally, to express the shape complexity, Shen (1992) and Shen et al. (1994) defined three normalized low-order moments that constitute the shape features, as follows:

$$
\begin{gathered}
F_{1}=\frac{\left(M_{2}\right)^{\frac{1}{2}}}{m_{1}}=\frac{\left\{\frac{1}{N} \sum_{n=0}^{N-1}\left[d(n)-m_{1}\right]^{2}\right\}^{\frac{1}{2}}}{m_{1}}, \\
F_{2}^{\prime}=\frac{\left(M_{3}\right)^{\frac{1}{3}}}{m_{1}}=\frac{\left\{\frac{1}{N} \sum_{n=0}^{N-1}\left[d(n)-m_{1}\right]^{3}\right\}^{\frac{1}{3}}}{m_{1}},
\end{gathered}
$$




$$
F_{3}^{\prime}=\frac{\left(M_{4}\right)^{\frac{1}{4}}}{m_{1}}=\frac{\left\{\frac{1}{N} \sum_{n=0}^{N-1}\left[d(n)-m_{1}\right]^{4}\right\}^{\frac{1}{4}}}{m_{1}}
$$

An additional shape descriptor, $m f=F_{3}^{\prime}-F_{1}$ is considered to show the roughness of a mass (Shen et al., 1994). The values of all of the shape features considered are limited to the range $[0,1]$.

Table 2 shows the shape features $\left(c f, F_{1}, F_{2}^{\prime}, F_{3}^{\prime}, m f\right)$ for the ALSSM, SSLS, MLACMLS and for the ground truth (expert), as well as $O V$. The values in this table are evaluated for all the images depicted in this paper, which have been selected from DDSM. As seen in Table 2, MLACMLS exhibits the maximum similarity $(O V)$ to the ground truth delineation for all cases, and exhibits highest correlation with ground truth shape features for 29 out of 35 values (83\%). For most of the cases in Table 2, $c f$ for the spiculated tumors is higher than the other kinds of the masses. Except for one case, the $m f$ values for MLACMLS, which act as a good indicator of shape roughness, are higher for spicules than other kinds of tumors. Considering the values of the shape features of the MLACMLS listed in table 2, we notice that there exists a noticeable difference between the corresponding shape feature values associated to the nonspiculated tumors (the first four study cases in table 2 for the MLACMLS) and those related to the spiculated ones (the last three study cases in table 2 for the MLACMLS). Therefore, we may conclude that the selected shape features can be shown to be robust features in a CAD system where we want to determine the type of a tumor, being either spiculated or non-spiculated. Further, the difference of features listed in Table 2 is small between the MLACMLS and the ALSSM in most of the cases where a non-spiculated tumor is segmented. The best way to compare these two methods is the $O V$ value (Overlap measure). Although the $O V$ values for these two methods are close, the MLACMLS outperforms the ALSSM in segmenting spiculated tumors, where the ALSSM fails to work properly as it is only designed to segment non-spiculated tumors. Note that the spiculated masses have higher probability to be a malignant tumor than the non-spiculated ones. 
Table 2

Quantitative results: the comparison between MLACMLS, ALSSM, SSLS and the ground truth (expert).

\begin{tabular}{cccccccc}
\hline Case & $O V$ & $c f$ & $F_{1}$ & $F^{\prime}{ }_{2}$ & $F^{\prime}$ & $m f$ & Method \\
\hline Figure & 0.7741 & 0.9225 & 0.2959 & 0.2369 & 0.3941 & 0.0982 & ALSSM \\
4(d) & 0.8467 & 0.8983 & 0.2962 & 0.2593 & 0.3985 & 0.1023 & MLACMLS \\
& & 0.9121 & 0.3059 & 0.2927 & 0.4160 & 0.1101 & EXPERT \\
\hline Figure & 0.8934 & 0.9127 & 0.2037 & 0.1624 & 0.2697 & 0.0660 & ALSSM \\
4(c) & 0.9123 & 0.8771 & 0.1807 & 0.1385 & 0.2379 & 0.0572 & MLACMLS \\
& & 0.8968 & 0.1911 & 0.1482 & 0.2449 & 0.0538 & EXPERT \\
\hline Figure & 0.6543 & 0.9504 & 0.2958 & 0.1994 & 0.3589 & 0.0630 & ALSSM \\
4(a) & 0.6733 & 0.9228 & 0.3233 & 0.1746 & 0.3972 & 0.0739 & MLACMLS \\
& & 0.9251 & 0.2262 & 0.1577 & 0.2805 & 0.0543 & EXPERT \\
\hline Figure & 0.7023 & 0.9492 & 0.2180 & 0.0789 & 0.2779 & 0.0599 & ALSSM \\
4(b) & 0.7710 & 0.9340 & 0.2219 & 0.0714 & 0.2812 & 0.0592 & MLACMLS \\
& & 0.9604 & 0.2843 & 0.0352 & 0.3477 & 0.0634 & EXPERT \\
\hline Figure & 0.4946 & 0.9900 & 0.3451 & 0.1592 & 0.4615 & 0.1164 & SSLS \\
7(a) & 0.7538 & 0.9614 & 0.3264 & 0.1296 & 0.4182 & 0.0918 & MLACMLS \\
& & 0.9526 & 0.2873 & 0.1217 & 0.3749 & 0.0876 & EXPERT \\
\hline Figure & 0.4933 & 0.9784 & 0.4139 & 0.2950 & 0.5347 & 0.1208 & SSLS \\
7(b) & 0.7564 & 0.9532 & 0.3047 & 0.2170 & 0.3928 & 0.0881 & MLACMLS \\
& & 0.9507 & 0.2778 & 0.2447 & 0.3754 & 0.0976 & EXPERT \\
\hline Figure & 0.6187 & 0.9818 & 0.4453 & 0.2911 & 0.5665 & 0.1212 & SSLS \\
7(c) & 0.7525 & 0.9400 & 0.3798 & 0.2247 & 0.4739 & 0.0941 & MLACMLS \\
& & 0.9489 & 0.3386 & 0.2011 & 0.4484 & 0.1098 & EXPERT \\
\hline
\end{tabular}

Note that the first four cases in the table are non-spiculated tumors and the latter three cases are spiculated one. The method to achieving the maximum similarity to the ground truth for all features of each study case is marked with a shaded cell.

Table 3 shows four morphological features (area, axis ratio, eccentricity, and perimeter length) as well as two statistical features (gray level mean and gray level std. dev.) applied for MLACMLS, ALSSM, SSLS, and expert over the same study cases in table 2. For each case, it shows that the area, the perimeter length, and the gray level std. dev. (APG) are the most robust features, showing higher feature difference between each method and the expert. The APGs may be alone applied for comparison between the MLACMLS, the ALSSM, and the SSLS or they are utilized along with the shape features from table 3. In either way, we have concluded that the MLACMLS, with having less overall difference to the feature values of the expert, outperforms the ALSSM and the SSLS. Note that in the case that the shape feature differences between either the MLACMLS and the expert or the ALSSM and the expert in table 2 is trivial, we also compare the methods using the feature difference between the APGs of each method and the expert from table 3. 
Table 3

Quantitative results: the comparison between MLACMLS, ALSSM, SSLS and the ground truth (expert) using morphological features and statistical features.

\begin{tabular}{cccccccc}
\hline \multicolumn{3}{c}{ Morphological features } & \multicolumn{4}{c}{ Statistical features } \\
\hline & $\begin{array}{c}\text { Area } \\
\text { (pixels) }\end{array}$ & $\begin{array}{c}\text { Axis } \\
\text { Ratio }\end{array}$ & Eccentricity & $\begin{array}{c}\text { Perimeter } \\
\text { length }\end{array}$ & $\begin{array}{c}\text { Gray level } \\
\text { mean }\end{array}$ & $\begin{array}{c}\text { Gray level } \\
\text { std. dev. }\end{array}$ & Method \\
\hline Figure & 23765 & 1.21 & 0.422 & 789.45 & 179.34 & 18.496 & SSLS \\
4(d) & 24532 & 1.12 & 0.442 & 813.17 & 184.82 & 18.243 & MLACMLS \\
& 25727 & 1.10 & 0.433 & 838.16 & 182.04 & 18.314 & EXPERT \\
\hline Figure & 37867 & 1.35 & 0.682 & 876.32 & 185.32 & 16.512 & ALSSM \\
4(c) & 32216 & 1.33 & 0.662 & 826.82 & 188.24 & 15.956 & MLACMLS \\
& 31893 & 1.26 & 0.614 & 771.02 & 192.74 & 13.252 & EXPERT \\
\hline Figure & 32054 & 1.31 & 0.671 & 887.45 & 198.32 & 16.76 & ALSSM \\
4(a) & 30465 & 1.29 & 0.632 & 898.67 & 200.01 & 18.898 & MLACMLS \\
& 36914 & 1.15 & 0.500 & 926.32 & 181.52 & 26.282 & EXPERT \\
\hline Figure & 25891 & 1.49 & 0.694 & 752.87 & 197.21 & 18.421 & ALSSM \\
4(b) & 28898 & 1.43 & 0.718 & 856.66 & 193.77 & 20.302 & MLACMLS \\
& 37481 & 1.16 & 0.514 & 844.03 & 180.57 & 22.130 & EXPERT \\
\hline Figure & 19948 & 1.48 & 0.736 & 1188.02 & 163.43 & 44.23 & SSLS \\
7(a) & 23325 & 1.75 & 0.797 & 1130.65 & 196.31 & 20.83 & MLACMLS \\
& 21954 & 1.85 & 0.842 & 1059.58 & 193.19 & 21.76 & EXPERT \\
\hline Figure & 22201 & 1.40 & 0.701 & 998.34 & 173.78 & 42.34 & SSLS \\
7(b) & 36261 & 1.05 & 0.291 & 113.72 & 150.49 & 29.61 & MLACMLS \\
& 36068 & 1.14 & 0.492 & 1571.37 & 150.94 & 31.62 & EXPERT \\
\hline Figure & 28530 & 1.10 & 0.43 & 1446.4 & 173.55 & 55.67 & SSLS \\
7(c) & 35083 & 1.24 & 0.68 & 1093.01 & 194.23 & 31.10 & MLACMLS \\
& 35122 & 1.37 & 0.59 & 1239.04 & 196.25 & 30.94 & EXPERT \\
\hline & & & & & & & \\
\hline
\end{tabular}

In addition, the $O V$ accuracy values for ALSSM, SSLS and MLACMLS calculated for 100 cases in our database, are presented in Fig. 9. The $O V$ measures for the MLACMLS are presented in two categories: The non-spiculated lesions and the spiculated ones (Figure 9). The maximum overlap value of 95\% is obtained using MLACMLS whereas the minimum overlap value of 51\% is obtained with SSLS. Fig. 9 shows that the SSLS achieves the lowest accuracy when delineating the spiculated tumors compared to other kinds of masses. Also, MLACMLS for all study images is more accurate than SSLS or ALSSM. 


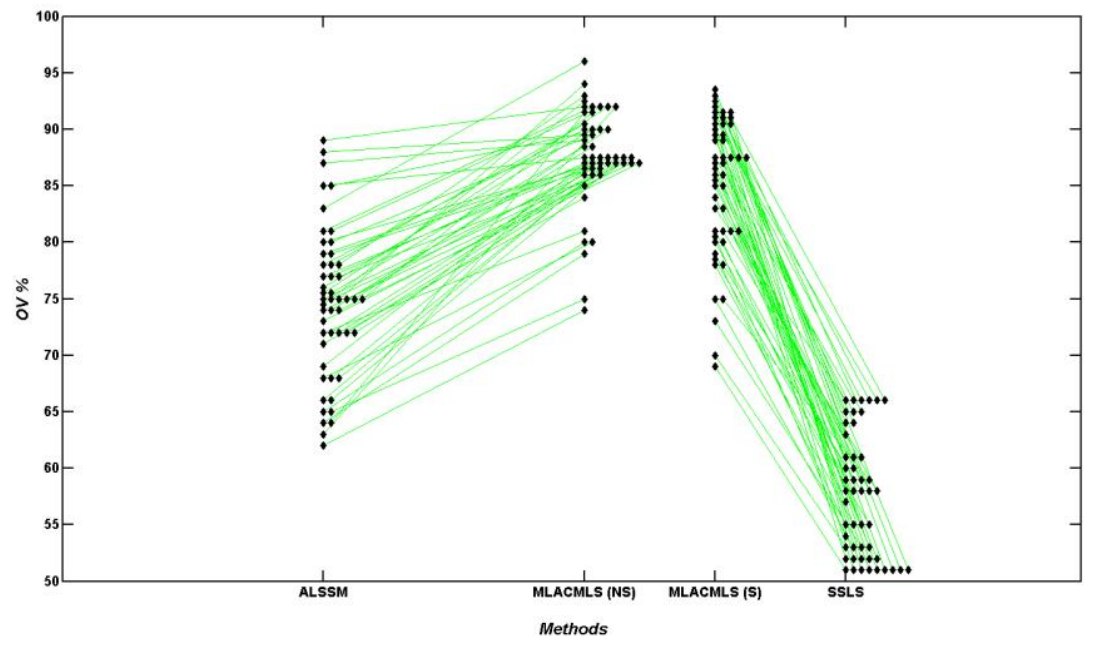

Fig. 9. The accuracy of the MLACMLS for delineating 100 images in our database in comparison with that of the ALSSM and the SSLS $(\beta=0.012)$. Note that the NS stands for the non-spiculated masses and the $\mathrm{S}$ stands for the spiculated ones.

To measure the interrater reliability measures, for every case study in our data base, we first calculated the overlap criterion between every two combination of the manually segmented images (M1, M2, and M3) as well as the overlap between the image of the proposed, automated segmentation method (AU) and the manual delineation. Then for each combination, the std. dev. of the overlap measures over all study cases in our database is calculated. Table 4 represents the results of the interrater reliability for each combination.

Table 4

Summary of interrater reliability measures.

\begin{tabular}{cccccccccccc}
\hline \multicolumn{2}{c}{ M1,M2 } & \multicolumn{2}{c}{ M2,M3 } & \multicolumn{2}{c}{ M1,M3 } & \multicolumn{2}{c}{ M1,AU } & \multicolumn{2}{c}{ M2,AU } & \multicolumn{2}{c}{ M3,AU } \\
\hline Mean & Std & Mean & Std & Mean & Std & Mean & Std & Mean & Std & Mean & Std \\
\hline 87.70 & 5.06 & 89.68 & 4.87 & 87.74 & 5.16 & 84.77 & 5.79 & 85.14 & 5.42 & 85.33 & 5.39 \\
\hline
\end{tabular}

Table 5 shows the mean overlap values achieved by ALSSM, SSLS and MLACMLS are respectively $74.32 \%, 57.11 \%$ and $86.85 \%$, calculated on the all our database. Also, the minimum std of 0.052 is achieved by MLACMLS. 
Table 5

Summary of segmentation overlap results.

\begin{tabular}{cccccc}
\hline Method & $\begin{array}{c}\text { Average of } \\
\boldsymbol{O} \boldsymbol{V}(\boldsymbol{\%})\end{array}$ & $\begin{array}{c}\text { Median of } \\
\boldsymbol{O} \boldsymbol{V}(\boldsymbol{\%})\end{array}$ & $\begin{array}{c}\text { Minimum of } \\
\boldsymbol{O} \boldsymbol{V}(\boldsymbol{\%})\end{array}$ & $\begin{array}{c}\text { Maximum of } \\
\boldsymbol{O} \boldsymbol{V}(\boldsymbol{\%})\end{array}$ & Std of $\boldsymbol{O} \boldsymbol{V}(\boldsymbol{\%})$ \\
\hline MLACMLS & 86.85 & 87 & 69 & 96 & 5.2314 \\
\hline ALSSM & 74.32 & 75.2 & 62 & 89 & 6.8575 \\
\hline SSLS & 57.11 & 58.1 & 51 & 66 & 5.9766 \\
\hline
\end{tabular}

Note that the results are averaged over all images in our database, and the best method is shaded in each column.

The average errors in shape feature values are summarized in Table 6. MLACMLS achieved the best results with an overall average error of 0.0299 and the least std. of 0.0171. In fact, MLACMLS has the lowest values for maximum error, median and std. of errors as compared to either ALSSM or SSLS. Also, the minimum error value of 0.0111 is less than SSLS (0.0281) and was almost equal to that of ALSSM (0.0098), which demonstrates that MLACMLS compares favorably with the competing methods.

Table 6

The average error of shape features.

\begin{tabular}{|c|c|c|c|c|c|c|c|c|c|c|}
\hline Method & $\begin{array}{l}\operatorname{Avg}^{*} \\
\Delta c f^{* *}\end{array}$ & $\begin{array}{l}\operatorname{Avg}^{*} \\
\Delta F_{1}^{* * *}\end{array}$ & $\begin{array}{c}\operatorname{Avg}^{*} \\
\Delta F_{2}{ }^{* * *}\end{array}$ & $\underset{\Delta \boldsymbol{F}_{3}}{\mathrm{Avg}^{* * *}}$ & $\begin{array}{c}\operatorname{Avg}^{*} \\
\Delta m f^{* *}\end{array}$ & $\begin{array}{c}\text { Overall } \\
\text { Avg* } \\
\text { Error }\end{array}$ & $\begin{array}{l}\text { Min. } \\
\text { Error }\end{array}$ & $\begin{array}{l}\text { Max. } \\
\text { Error }\end{array}$ & $\begin{array}{c}\text { Median } \\
\text { Error }\end{array}$ & $\begin{array}{c}\text { Std } \\
\text { Error }\end{array}$ \\
\hline MLACMLS & 0.0116 & 0.0440 & 0.0388 & 0.0440 & 0.0111 & 0.0299 & 0.0111 & 0.0440 & 0.0388 & 0.0171 \\
\hline$\overline{\text { ALSSM }}$ & 0.0147 & 0.0416 & 0.0428 & 0.0517 & 0.0098 & 0.0321 & 0.0098 & 0.0517 & 0.0416 & $\overline{0.0186}$ \\
\hline SSLS & 0.0337 & 0.1302 & 0.0597 & 0.1323 & 0.0281 & 0.0768 & 0.0281 & 0.1323 & 0.0597 & 0.0511 \\
\hline
\end{tabular}

Note that the method with the minimum Error is shaded in each column.

${ }^{*}$ Avg $=$ Average.

${ }^{* *} \Delta \mathbf{x}=\mathbf{x}$ (Method) $-\mathbf{x}($ Expert $)$.

The correlation coefficient between the shape features of each method and the same shape factor of the ground truth as well as the statistical significance are presented in Table 7. All the image data in our database are considered in this Table. The highest correlation coefficients are achieved by MLACMLS in comparison to the other methods. The maximum correlation coefficient of the MLACMLS, ALSSM and SSLS are 93.21\%, 90.78\%, and 89.05\%, and their minimum correlation coefficient are $70.54 \%, 41.34 \%$, and $54.22 \%$, respectively. MLACMLS achieved the minimum variability in the correlation values (less than 23\%). Our results show that the performance of the methods is statistically significant with $p$ values less than 0.05 . 


\section{Table 7}

Computing the correlation coefficient between shape factors of each method and the same shape factors of the ground truth segmentation, performed over the whole of our database.

\begin{tabular}{|c|c|c|c|c|c|c|c|c|c|c|}
\hline \multirow[t]{2}{*}{ Method } & \multicolumn{2}{|c|}{ cf, Exp" } & \multicolumn{2}{|c|}{$F_{1}, \mathbf{E x p}^{*}$} & \multicolumn{2}{|c|}{$\mathbf{F}_{2}^{\prime}, \mathbf{E x p}^{*}$} & \multicolumn{2}{|c|}{$\mathbf{F}_{3}^{\prime}, \mathbf{E x p}^{*}$} & \multicolumn{2}{|c|}{ mf, Exp" } \\
\hline & $\vec{\rho}$ & $\mathbf{S S}^{*{ }^{* 3}}$ & $\bar{\rho}$ & $\mathbf{S S}^{* *^{*}}$ & $\vec{\rho}$ & $\mathbf{S S}^{\sin }$ & $\bar{\rho}$ & $\mathbf{S S}^{\text {with }}$ & $\bar{\rho}$ & $\mathbf{S S}^{* *}$ \\
\hline MLACMLS & 0.9234 & $2 \mathrm{e}-5$ & 0.7241 & $7 e-5$ & 0.9321 & $1 \mathrm{e}-5$ & 0.7054 & $8 \mathrm{e}-5$ & 0.9123 & $3 e-5$ \\
\hline ALSSM & 0.8023 & $1 \mathrm{e}-4$ & 0.4134 & $5 \mathrm{e}-4$ & 0.9078 & $4 e-5$ & 0.5431 & $3 e-4$ & 0.8861 & $4 e-5$ \\
\hline SSLS & 0.6235 & $4 \mathrm{e}-4$ & 0.5986 & $4 \mathrm{e}-4$ & 0.8905 & $9 e-5$ & 0.5422 & $3 e-4$ & 0.8712 & $6 e-5$ \\
\hline
\end{tabular}

Note that the method with the maximum correlation coefficient is shaded in each column.

${ }^{*} \mathbf{E x p}=$ Expert.

${ }^{* *} \boldsymbol{\rho}=$ Correlation coefficient.

\section{* SS= Statistical Significance.}

To investigate the effect of the FCLAHE on the accuracy of the segmentation with MLACMLS, we present, in Fig. 10, the $O V$ values for each study case with and without FCLAHE. We note an increase in the overlap with expert segmentation by around $34 \%$ (from $52.23 \%$ to $86.85 \%$ ) when FCLAHE was employed (averaged over 100 images), compared to a 16.3\% increase when using FCLAHE for CSM. The above results support the conclusion of our previous work (Rahmati et al., 2010) which stated that FCLAHE works better for a PDF-based segmentation algorithm, such as MLACMLS.

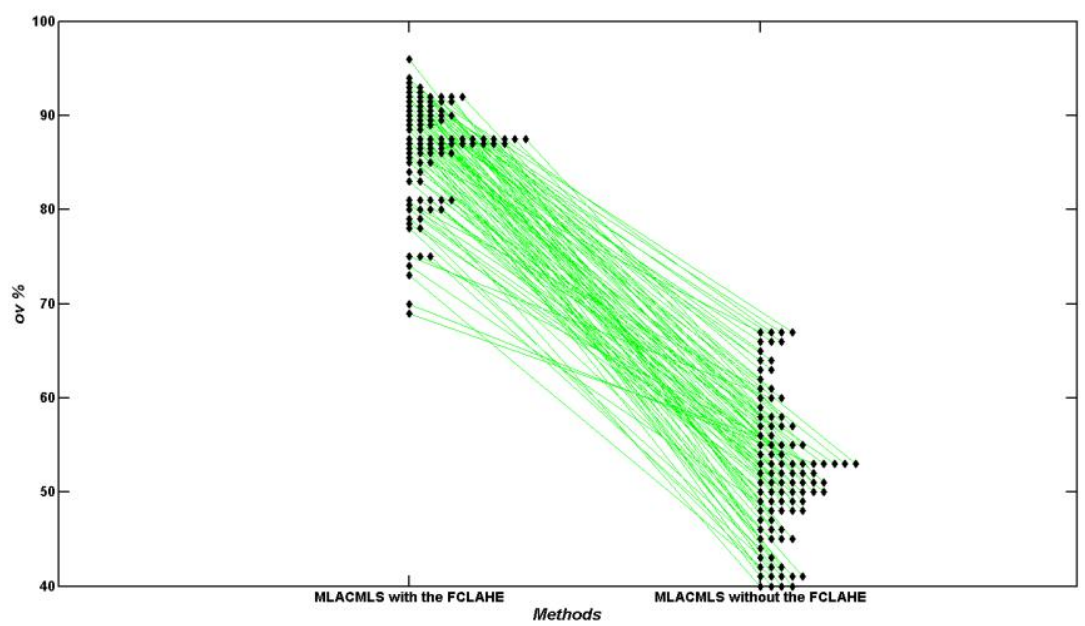

Fig. 10. The effect of the FCLAHE on the accuracy $(O V)$ of the MLACMLS. 


\subsection{Method's robustness and sensitivity to parameters}

\subsubsection{MLACMLS robustness with respect to seed point location}

In this sub-section, the robustness of MLACMLS is evaluated against varying the location of the seed point selected by the user. Various seed points are selected on a radial line connecting two seed points: one at the center of the mass and the other at the mass boundary, i.e. gradually moving away from the core of the mass towards the boundary. Fig. 11 shows two sample seed points and the corresponding segmentation. We considered the worst state of the segmentation accuracy for each image when the seed points are changed. The mean (over the 100 images) and the std of the worst state of the segmentation accuracy calculated on our database were 79.1 and 7.12, respectively. Also, the maximum segmentation error for changing seed point computed on our image database was less than $10 \%$.

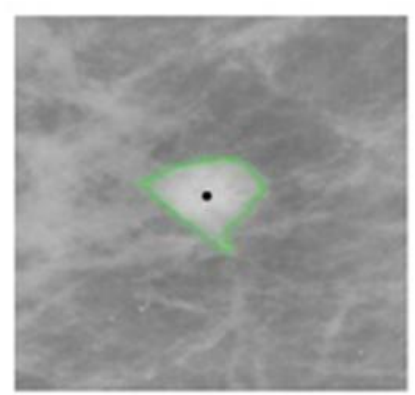

(a)

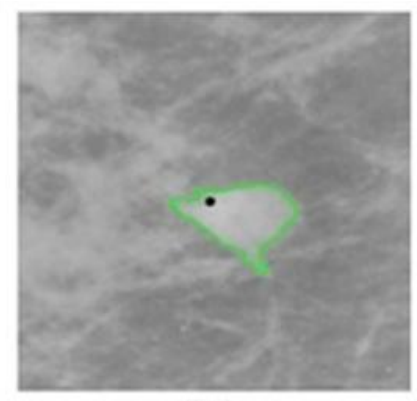

(b)

Fig. 11. Example of the MLACMLS performance when two different seed points are used to initialize the segmentation of the same image. (a) The seed point (black dot inside the mass) is placed in near the mass core. (b) The seed point (black dot) is placed near the boundary of the mass. Note that the images are already filtered by the FCLAHE.

\subsubsection{Robustness to changes in FCLACHE's openness parameter}

As previously mentioned, prior to using MLACMLS, we use the FCLAHE filter and set its openness parameter $(\beta)$ to 0.012 . Although, this parameter is strictly a parameter of FCLAHE and not of 
the proposed MLACMLS, we assess the robustness of MLACMLS to changes in this pre-processing parameter. Our experiences indicate that appropriate values of $\beta$ are in the range [0.01, 0.02]. Fig. 12 depicts the final delineation contour of MLACMLS for the minimum and the maximum value of $\beta$ for two mammographic images chosen from the two different data bases (DDSM and the images reproduced from Guliato et al. (2003a, 2003b)). Fig. 12 shows negligible difference between the final segmentations. For higher $\beta$ values, the segmentation algorithm tends to over-segment. Fig. 12(a) with low contrast is slightly more sensitive to $\beta$ variations than Fig. 12(c) with high contrast.

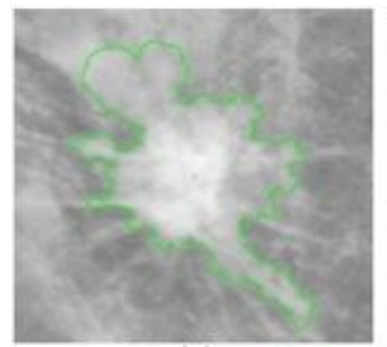

(a)

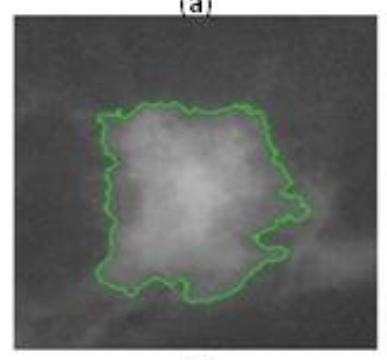

(c)

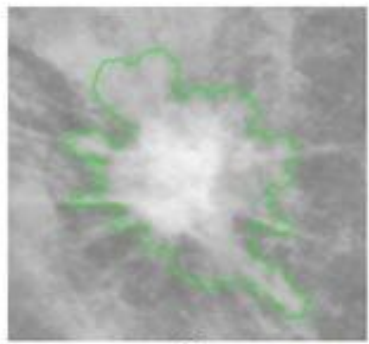

(b)

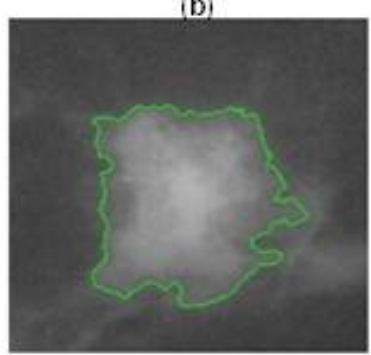

(d)

Fig. 12. MLACMLS robustness with respect to the choice of $\beta$ for two mammographic images that contain spiculated tumors. The final segmented image with $\beta$ value of 0.01 is shown in (a) and 0.02 in (b) performed on a low contrast image of DDSM. The final contour of MLACMLS with $\beta$ value of 0.01 in (c) and 0.02 in (d) for the image reproduced with permission from Guliato et al. (2003b), with higher contrast compared to that in of DDSM.

The mean and the std of the worst segmentation accuracy $(O V)$ for changing values of $\beta$ with a step size of 0.001 in the range of $[0.01,0.02]$ computed on our database were 77.1 and 6.61 , respectively. The maximum change of $O V$ value was less than $12 \%$ as $\beta$ changed by $100 \%$ (from 0.01 to 0.02 ). 


\subsection{Modeling mammograms by other distributions}

In this sub-section, we assess the performance of our method when using probability distributions other than the Gamma distribution for modeling the variation of gray levels of mammographic image. In particular, we examine the performance of MLACMLS when using the Rayleigh distribution and the Poisson distribution (Rahmati and Ayatollahi, 2009). The final results of applying Rayleigh distribution to achieve the ML segmentation, detailed in section 3.3, are depicted in Fig. 13 for a spiculated tumor and for a non-spiculated tumor in Fig. 14. Note that, in Fig. 14, there is no convergence using this distribution and the contour tends to get bigger and bigger with iteration number. In Rahmati and Ayatollahi (2009), we applied the Poisson distribution to segment breast lesion, and we achieved an average segmentation accuracy of $81 \%$, compared to $86.85 \%$ in this work using the Gamma distribution.
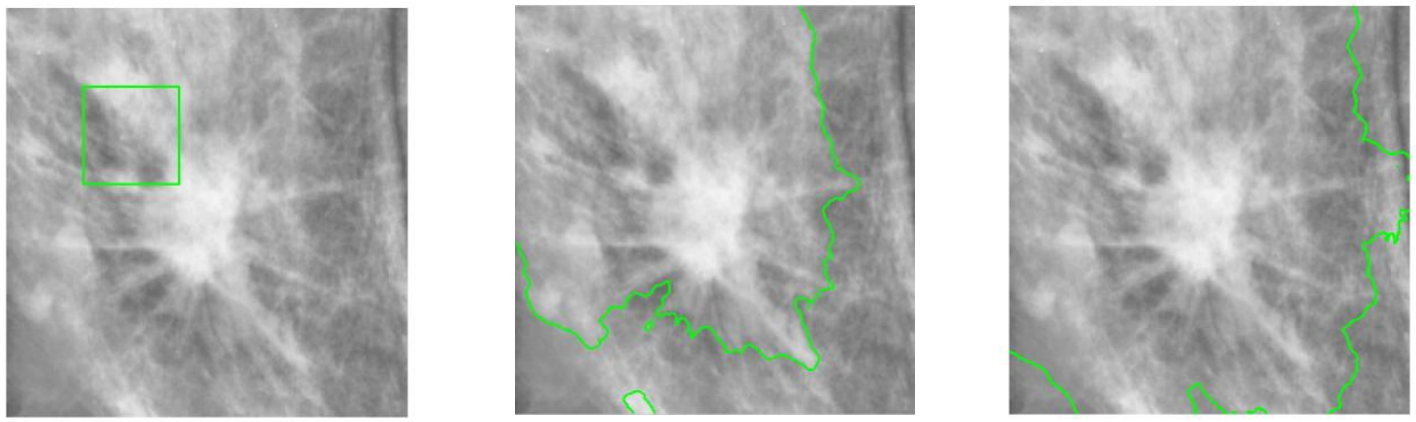

Fig. 13. Segmentation of a spiculated tumor from DDSM database with the assumption of Rayleigh distribution to evaluate the ML segmentation: From left to right columns, initial contour, the evolved contour in the iteration 56 and the resulting contour in iteration 171. Note that the contour continues to get bigger and bigger for higher iteration numbers and does not converge. The segmenttaion of the same image using Gamma distribution is shown in Fig. 7(a). Note that the original image is filtered by the FCLAHE. 

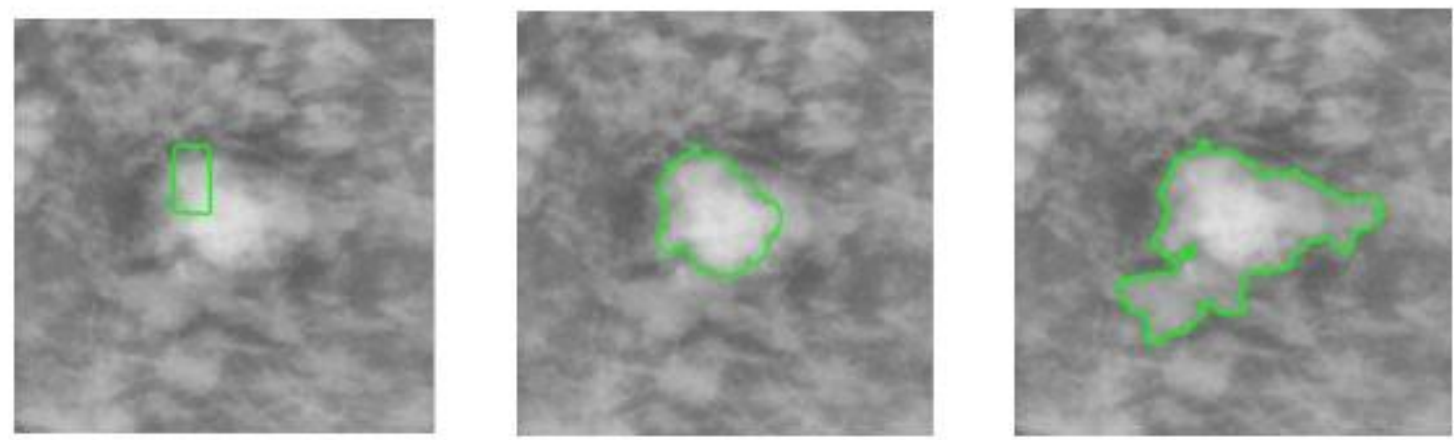

Fig. 14. Segmentation of a non-spiculated tumor from DDSM database with the assumption of Rayleigh distribution to evaluate the ML segmentation: From left to right columns, initial contour, iteration 55 and iteration 175. The segmenttaion of the same image using Gamma distribution is shown in Fig. 4(a). Note that the original image is filtered by the FCLAHE.

In Fig. 15, we show the accuracy value of the MLACMLS for 100 images of our database when using three distributions: Rayleigh, Poisson, and Gamma. Comparing the competing distributions, the assumption of Gamma distribution offers higher segmentation accuracy for most of 100 case studies.

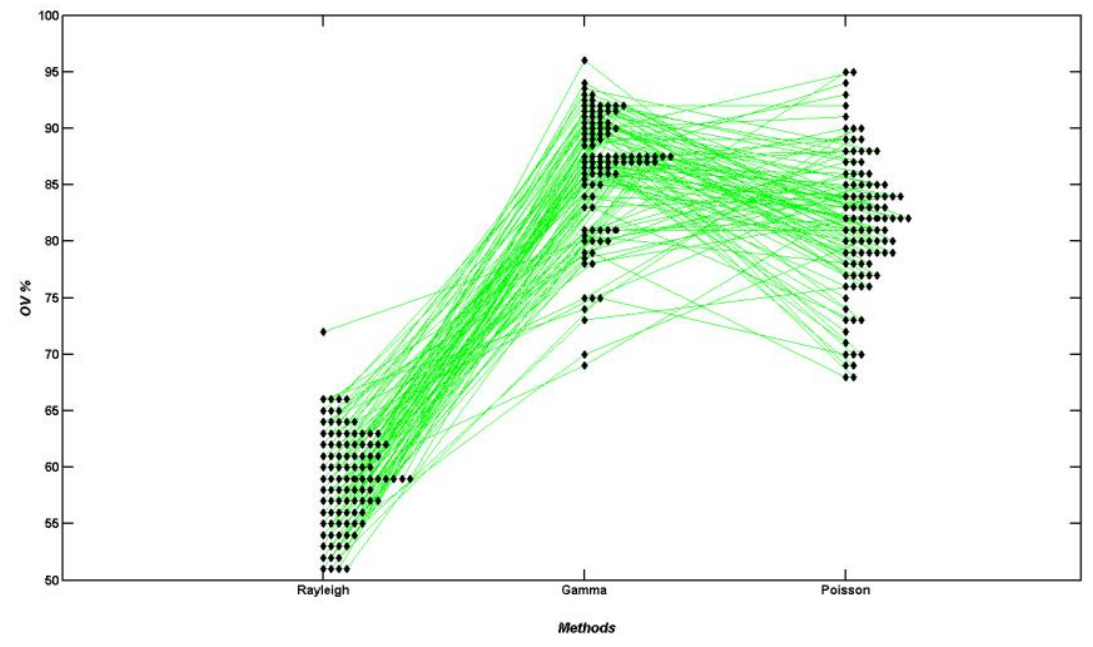

Fig. 15. The comparison of the performance of our proposed method when using three different exponential distributions. In every three statistical distributions, the openness value $(\beta)$ was set to a fixed value of 0.012 . 


\subsection{Evaluating KL divergence metric}

In this sub-section, we compare the suitability of using the KL divergence vs. the proposed log-likelihood as the objective function that drives the contour evolution for mammography segmentation. The goal here is to evaluate which objective function correlates best with the correctness of the segmentation. Ideally, the objective function should increase monotonically as the segmentation improves. We selected 10 study cases from our database, and for each selected case, we captured 10 segmented images during the iterations. Then, the KL divergence between the foreground and background PDFs was calculated and compared to the proposed Log Likelihood function for each segmented image. Figure 16 shows the results for the study case in Fig. 5. As shown in Figure 16, the KL divergence does not show a monotonic increase in as the segmentation improves (the overlap with ground truth increases), whereas the proposed Log Likelihood function shows a monotonic increase. The profile of the curves for the other 9 study cases was similar to that of Fig. 16 (a) and (b), meaning a monotonic increase for the proposed Log Likelihood function and a dominant maximum local peak involved for the KL divergence. This suggests that the proposed Log Likelihood function is more robust objective function for mammography segmentation. The maximum KL divergence value (dominant maximum peak) averaged over the 10 study cases was 22.27 and occurred in an averaged overlap value of 57.74. The error bars in Figure 16(a) shows the maximum std of 3.72 occurring at the maximum KL divergence value. In Figure 16(b), the changes in the std values over the overlap values are not noticeable, a trait absent in the KL divergence plot. Fig. 16(c) shows the values of the energy function of the ACWOE for the same study case in Fig. 5. The energy function of the ACWOE tends to increase as the segmentation improves, producing higher error bars when comparing with the KL divergence (Fig. 16(a)) and the MLACMLS (Fig.16(b)). 


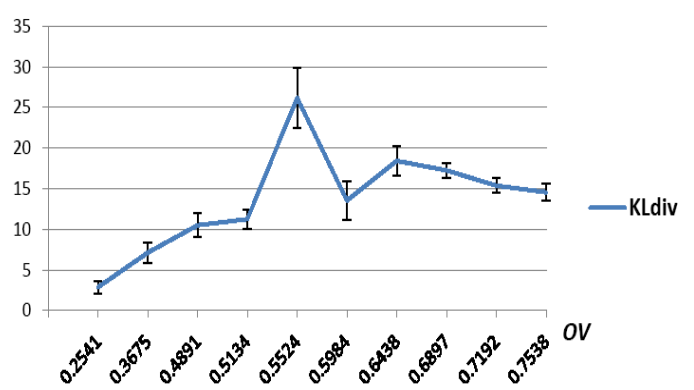

(a)

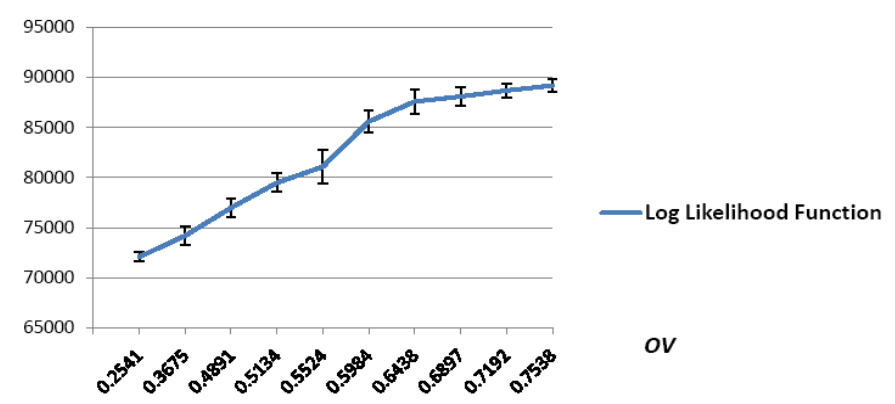

(b)

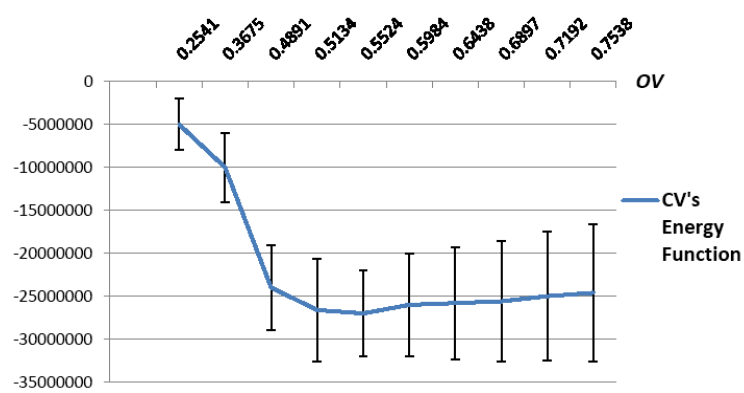

(c)

Fig. 16. Comparison of KL-divergence vs. log-likelihhod as the objective function driving the segmentation. (a) The KL divergence values as a function of $O V$ over 10 segmented images. (b) The values for the proposed Log Likelihood function as a function of $O V$ for the same segmented images in (a)(Note how the Log-likelihood monotonically increases as the segmentation improves, a trait absent in the KL-divergence plot). (c) The values for the energy function of the ACWOE as a function of $O V$ for the same images in (a) when the best initialization of tiled is set up for all the 10 images.

\section{Discussion and conclusions}

We proposed a novel level set active contour model, referred to as MLACMLS, specialized for the delineation of lesions in digital mammograms with a focus on spicules, which are the most important sign of malignancy for a mass without any biopsy. Our curve evolution approach maximizes a likelihood function that favors dividing the image into two maximally homogeneous areas; the foreground and background. The Gamma distribution's estimated parameters for both the foreground and background are updated automatically with every iteration, using only integral quantities of the image. Therefore, our proposed ML segmentation is able to deal with noisy images without clear edges and with interior and exterior regions following the same family of distributions albeit with different and unknown parameters. The proposed computer-aided mammography delineation method incorporates the advantages of the level 
set and region-based active contour model of Chan-Vese (2001). It includes noise robustness capability because of taking advantage of integrals of the image under investigation. The method requires the specification of a single seed point. The proposed model was evaluated on mammogram images and compared quantitatively and qualitatively with the state of the art approaches, ALSSM and SSLS models, on real mammographic images.

Our results show that MLACMLS is superior to SSLS and to ALSSM in the delineation of different types of tumors (with an average accuracy of $86.85 \%$ for MLACMLS vs. $57.11 \%$ for SSLS and 74.32\% for ALSSM). In our previous publication (Rahmati and Ayatollahi, 2009), we had already applied the CSM, a threshold based segmentation method, on the same data base and concluded that the level set based algorithm using the Poisson distribution works better than the CSM for mammography segmentation. Furthermore, in this work, we show how our proposed level set based segmentation algorithm using the Gamma distribution outperforms the one using Poisson distribution, meaning it outperforms the CSM as well. Further, in Fig. 8 and Fig. 16 (c), we show how our proposed segmentation method outperforms the ACWOE of Chan and Vese (2001). Note that as we are using the DDSM database, which does not offer two independent scans from the same patient, we are not able to measure the reproducibility.

Our update equation is essentially a form of a gradient ascent (based on Euler-Lagrange equation) and the Gradient ascent will eventually converge to a local maxima (aside from saddle points). Our results show that the proposed segmentation algorithm converged to a local maximum of the likelihood function for all the study cases in our data base (100 images). As fig. 5(c) shows for one study case, the algorithm converged to a local maximum after 500 iterations. Similar convergence results were achieved for all other study cases in our data set. The fourth row of Fig. 8 represents the proposed likelihood function converging to a local maximum for different initializations. 
Different imaging systems result in different intensity variations for mammograms. For example, fig. 3 in our paper shows an imaging system that produces a big tail in the histogram of the mammogram, deviating from being a Gaussian distribution. However, we have a shorter tail in the other mammogram shown in Fig. 5(b) produced by a different imaging system than the one in fig. 3. The benefit of using the proposed model, compared to that of Paragios's method, is that ours is more capable in adapting to different imaging systems having different parameters. The adaptation comes from the incorporation of the Gamma distribution, having two tunable parameters (scale factor and shape factor), into the energy function. Moreover, the proposed scale factors in equations A5 and A6, in the appendix A, are weighted scale factors by the shape factors ( $k i$ and $k e$ ), enabling the segmentation algorithm to adapt the estimated intensity distribution to the specific gray level intensity variations across all 100 tested images. For example, the weighted scale factors help the segmentation algorithm to adapt to the intensity changes due to different setting for the $\mathrm{x}$-ray imaging system. In the special state of having $k i=k e=1$, we achieve nonweighted scale factors, which are equivalent to the proposed scale factors by Ayed et al. (2005).

Our experiments showed that adapting the two-parameter Gamma distribution gives better results compared to two other possible distributions: the one-parameter Rayleigh distribution and the Poisson distribution (section 4.4). We note that the original noise statistics of the mammography image is transformed by applying the FCLAHE and the Gamma distribution is considered based on the empirical observation following the results of the FCLAHE.

Moreover, our results showed better correlation between the ML-based objective function and the segmentation accuracy, when compared to the KL divergence based objective function (Georgiou et al., 2007). 


\section{Appendix A: Optimal PDF parameters estimation}

In this appendix, we present how to estimate the optimal parameters of the PDF with a ML approach. The Gamma probability function is defined by:

$P(I(x, y))=I(x, y)^{k-1} \frac{e^{\frac{-I(x, y)}{\theta}}}{\theta^{k} \Gamma(k)} \quad, I(x, y)>0, \quad \theta, k>0$,

where $k$ is the shape factor, $\theta$ is the scale factor and $I(x, y)$ takes discrete values. Let $k_{i}$ and $k_{e}$ be the shape parameter values for the foreground and for the background pixels, respectively. Also $\theta_{i}$ and $\theta_{e}$ be the scale parameter values for the target and for the background pixels. Therefore the log-likelihood is:

$l\left(I, C, \theta_{i}, \theta_{e}, k_{i}, k_{e}\right)=\log \left(\prod_{(x, y) \in \Omega} P(I(x, y))\right)=$

$\left(k_{i}-1\right) \sum_{(x, y) \in \Omega_{i}} \log (I(x, y))-\sum_{(x, y) \in \Omega_{i}}\left(\frac{I(x, y)}{\theta_{i}}\right)-A_{i} k_{i} \log \left(\theta_{i}\right)-A_{i} \log \left(\Gamma\left(k_{i}\right)\right)+$

$\left(k_{e}-1\right) \sum_{(x, y) \in \Omega_{e}} \log (I(x, y))-\sum_{(x, y) \in \Omega_{e}}\left(\frac{I(x, y)}{\theta_{e}}\right)-A_{e} k_{e} \log \left(\theta_{e}\right)-A_{e} \log \left(\Gamma\left(k_{e}\right)\right)$,

where $A_{i}$ and $A_{e}$ are the number of pixels of the foreground and of the background. To obtain the ML estimate of the parameters $\theta_{i}$ and $\theta_{e}$, we calculate the first derivative of $l\left(I, C, k_{i}, k_{e}, \theta_{i}, \theta_{e}\right)$ with respect to $\theta_{i}$ :

$$
\frac{\partial l}{\partial \theta_{i}}=\sum_{(x, y) \in \Omega_{i}}\left(\frac{I(x, y)}{\theta_{i}{ }^{2}}\right)-\frac{A_{i} k_{i}}{\theta_{i}}=0,
$$

and to $\theta_{e}$ :

$$
\frac{\partial l}{\partial \theta_{e}}=\sum_{(x, y) \in \Omega_{e}}\left(\frac{I(x, y)}{\theta_{e}{ }^{2}}\right)-\frac{A_{e} k_{e}}{\theta_{e}}=0 .
$$

So, the optimal values for these two parameters can be written as:

$$
\theta_{i}=\frac{1}{A_{i} k_{i}} \sum_{(x, y) \in \Omega_{i}} I(x, y),
$$

and:

$$
\theta_{e}=\frac{1}{A_{e} k_{e}} \sum_{(x, y) \in \Omega_{e}} I(x, y) .
$$


Now, to estimate the parameters $k_{i}$ and $k_{e}$, we eliminate the estimated parameters in above in function $l$ by introducing them back in the log-likelihood:

$$
\begin{aligned}
& l\left(I, C, \theta_{i}, \theta_{e}, k_{i}, k_{e}\right)=\left(k_{i}-1\right) \sum_{(x, y) \in \Omega_{i}} \log (I(x, y))-A_{i} k_{i}-A_{i} k_{i} \log \left(\frac{\sum_{(x, y) \in \Omega_{i} I} I(x, y)}{A_{i} k_{i}}\right)- \\
& A_{i} \log \left(\Gamma\left(k_{i}\right)\right)+\left(k_{e}-1\right) \sum_{(x, y) \in \Omega_{e}} \log (I(x, y))-A_{e} k_{e}-A_{e} k_{e} \log \left(\frac{\sum_{(x, y) \in \Omega_{e}} I(x, y)}{A_{e} k_{e}}\right)-A_{e} \log \left(\Gamma\left(k_{e}\right)\right) .
\end{aligned}
$$

As before, the ML estimate of the parameters $k_{i}$ and $k_{e}$ is obtained by:

$$
\begin{aligned}
& \frac{\partial l}{\partial k_{i}}=0 \stackrel{\text { yields }}{\longrightarrow} \log \left(k_{i}\right)-\Psi\left(k_{i}\right)=\log \left(\frac{1}{A_{i}} \sum_{(x, y) \in \Omega_{i}} I(x, y)\right)-\frac{1}{A_{i}} \sum_{(x, y) \in \Omega_{i}} \log (I(x, y)), \\
& \frac{\partial l}{\partial k_{e}}=0 \stackrel{\text { yields }}{\longrightarrow} \log \left(k_{e}\right)-\Psi\left(k_{e}\right)=\log \left(\frac{1}{A_{e}} \sum_{(x, y) \in \Omega_{e}} I(x, y)\right)-\frac{1}{A_{e}} \sum_{(x, y) \in \Omega_{e}} \log (I(x, y)),
\end{aligned}
$$

where $\Psi(k)$ is Digamma function defined as:

$$
\Psi(k)=\frac{\dot{\Gamma}(k)}{\Gamma(k)}
$$

in which $\Gamma$ is the Gamma function. To summarize the above equations for reaching to the estimated values for $k_{i}$ and $k_{e}$, we define two new parameters as $S_{\mathrm{i}}$ and $\mathrm{S}_{\mathrm{e}}$ :

$S_{i}=\log \left(\frac{1}{A_{i}} \sum_{(x, y) \in \Omega_{i}} I(x, y)\right)-\frac{1}{A_{i}} \sum_{(x, y) \in \Omega_{i}} \log (I(x, y))$,

$S_{e}=\log \left(\frac{1}{A_{e}} \sum_{(x, y) \in \Omega_{e}} I(x, y)\right)-\frac{1}{A_{e}} \sum_{(x, y) \in \Omega_{e}} \log (I(x, y))$.

Choi and Wette have shown an equivalent term as (Choi and Wette, 1969):

$$
\log (k)-\Psi(k) \approx \frac{1}{k}\left(\frac{1}{2}+\frac{1}{12 k+2}\right) \approx \frac{1}{2 k} \quad \text { if } \quad k \gg 0 .
$$

By introducing the above term back in the expressions $\frac{\partial l}{\partial k_{i}}$ and $\frac{\partial l}{\partial k_{e}}$, we obtain the optimal values for $k_{i}$ and $k_{e}$ as follows:

$$
\begin{aligned}
k_{i} & \approx \frac{1}{2 S_{i}}, \\
k_{e} & \approx \frac{1}{2 S_{e}} .
\end{aligned}
$$

By replacing these estimates into the expression $l$, we achieve: 
$l_{\text {opt }}\left(I, C, \theta_{i}, \theta_{e}, k_{i}, k_{e}\right)=$

$-\sum_{(x, y) \in \Omega_{i}} \log (I(x, y))-A_{i} k_{i} S_{i}+A_{i} k_{i}\left(\log \left(k_{i}\right)-1\right)-A_{i} \log \left(\Gamma\left(k_{i}\right)\right)-\sum_{(x, y) \in \Omega_{e}} \log (I(x, y))-$

$A_{e} k_{e} S_{e}+A_{e} k_{e}\left(\log \left(k_{e}\right)-1\right)-A_{e} \log \left(\Gamma\left(k_{e}\right)\right)$

Simplifying the above, we arrive at:

$l_{\text {opt }}\left(I, C, \theta_{i}, \theta_{e}, k_{i}, k_{e}\right)=$

$-\sum_{(x, y) \in \Omega} \log (I(x, y))-\frac{N}{2}-\frac{A_{i}}{2 S_{i}}\left(\log \left(2 S_{i}\right)+1\right)-A_{i} \log \left(\Gamma\left(\frac{1}{2 S_{i}}\right)\right)-\frac{A_{e}}{2 S_{e}}\left(\log \left(2 S_{e}\right)+1\right)-$

$A_{e} \log \left(\Gamma\left(\frac{1}{2 S_{e}}\right)\right)$

where $N$ is the total number of pixels. All the terms which do not depend on the partition $\Omega_{i}$ and $\Omega_{e}$ can be omitted. Finally we arrive at the expression used in (5):

$L_{\text {opt }}\left(I, C, \theta_{i}, \theta_{e}, k_{i}, k_{e}\right)=$

$-\frac{A_{i}}{2 S_{i}}\left(\log \left(2 S_{i}\right)+1\right)-A_{i} \log \left(\Gamma\left(\frac{1}{2 S_{i}}\right)\right)-\frac{A_{e}}{2 S_{e}}\left(\log \left(2 S_{e}\right)+1\right)-A_{e} \log \left(\Gamma\left(\frac{1}{2 S_{e}}\right)\right)$.

\section{Appendix B: Energy function minimization}

In this appendix, we explain how to minimize the energy function in (9) by deriving the EulerLagrange equations and the related flow. To calculate the first variation of the energy function with respect to $\Phi$, which its dependency on $\mathrm{x}$ and y has been dropped for clarity, the following equation has to solve:

$$
\lim _{t \rightarrow 0} \frac{1}{t}(F(\Phi+t \psi)-F(\Phi))=0 .
$$

where $\psi$ is of the same type as $\Phi$. To compute this variation, we consider the regularized version of the Heaviside function $H$ and of the Dirac function $(\delta)$ as $H_{\varepsilon}$ and $\delta_{\varepsilon}$, as follows (Chan and Vese, 2001):

$$
\begin{aligned}
& H_{\varepsilon}(x)=\frac{1}{2}\left(1+\frac{2}{\pi} \arctan \left(\frac{x}{\varepsilon}\right)\right), \\
& \delta_{\varepsilon}(x)=\frac{d}{d x} H_{\varepsilon}(x)=\frac{1}{\pi} \cdot \frac{\varepsilon}{\left(\varepsilon^{2}+x^{2}\right)} .
\end{aligned}
$$


The expressions $S_{i}$ and $S_{\mathrm{e}}$ (cf. (A11-12)) and their variations in respect to $\Phi$ can be written using the Heaviside function $H$ as:

$$
\begin{aligned}
& S_{i}=\log \left(\frac{\int_{\Omega} I H_{\varepsilon}(\Phi) d x d y}{\int_{\Omega} H_{\varepsilon}(\Phi) d x d y}\right)-\frac{\int_{\Omega} \log (I) H_{\varepsilon}(\Phi) d x d y}{\int_{\Omega} H_{\varepsilon}(\Phi) d x d y}, \\
& S_{e}=\log \left(\frac{\int_{\Omega} I\left(1-H_{\varepsilon}(\Phi)\right) d x d y}{\int_{\Omega}\left(1-H_{\varepsilon}(\Phi)\right) d x d y}\right)-\frac{\int_{\Omega} \log (I)\left(1-H_{\varepsilon}(\Phi)\right) d x d y}{\int_{\Omega}\left(1-H_{\varepsilon}(\Phi)\right) d x d y} . \\
& \left.\frac{\partial}{\partial t} S_{i}(\Phi+\psi t)\right|_{t=0}=\sigma S_{i}= \\
& \int_{\Omega} \delta_{\varepsilon}(\Phi)\left[\frac{I \int_{\Omega} H_{\varepsilon}(\Phi) d x d y-\int_{\Omega} I H_{\varepsilon}(\Phi) d x d y}{\int_{\Omega} H_{\varepsilon}(\Phi) d x d y \times \int_{\Omega} I H_{\varepsilon}(\Phi) d x d y}-\right.
\end{aligned}
$$$$
\left.\frac{\partial}{\partial t} S_{i}(\Phi+\psi t)\right|_{t=0}=\sigma S_{i}=
$$$$
\left.\frac{\log (I) \int_{\Omega} H_{\varepsilon}(\Phi) d x d y-\int_{\Omega} \log (I) H_{\varepsilon}(\Phi) d x d y}{\left(\int_{\Omega} H_{\varepsilon}(\Phi) d x d y\right)^{2}}\right] \psi d x d y
$$

replacing $\int_{\Omega} H_{\mathcal{E}}(\Phi) d x d y, \int_{\Omega} I H_{\mathcal{E}}(\Phi) d x d y$, and $\int_{\Omega} \log (I) H_{\mathcal{E}}(\Phi) d x d y$ by three constant variables named $c_{i 1}, c_{i 2}$, and $c_{i 3}$; respectively, we can write:

$\left.\frac{\partial}{\partial t} S_{i}(\Phi+\psi t)\right|_{t=0}=\sigma S_{i}=\int_{\Omega} \delta_{\varepsilon}(\Phi)\left[\frac{I c_{i 1}-c_{i 2}}{c_{i 1} c_{i 2}}-\frac{\log (I) c_{i 1}-c_{i 3}}{\left(c_{i 1}\right)^{2}}\right] \psi d x d y=\int_{\Omega} \delta_{\varepsilon}(\Phi)\left[C_{i}\right] \psi d x d y$,

where $C_{i}$ is an auxiliary vector, and:

$$
\begin{aligned}
& \left.\frac{\partial}{\partial t} S_{e}(\Phi+\psi t)\right|_{t=0}=\sigma S_{e}= \\
& \int_{\Omega} \delta_{\varepsilon}(\Phi)\left[\frac{-I \int_{\Omega}\left(1-H_{\varepsilon}(\Phi)\right) d x d y+\int_{\Omega} I\left(1-H_{\varepsilon}(\Phi)\right) d x d y}{\int_{\Omega}\left(1-H_{\varepsilon}(\Phi)\right) d x d y \times \int_{\Omega} I\left(1-H_{\varepsilon}(\Phi)\right) d x d y}+\right. \\
& \left.\frac{\log (I) \int_{\Omega}\left(1-H_{\varepsilon}(\Phi)\right) d x d y-\int_{\Omega} \log (I)\left(1-H_{\varepsilon}(\Phi)\right) d x d y}{\left(\int_{\Omega}\left(1-H_{\mathcal{E}}(\Phi)\right) d x d y\right)^{2}}\right] \psi d x d y
\end{aligned}
$$

replacing $\int_{\Omega}\left(1-H_{\varepsilon}(\Phi)\right) d x d y, \int_{\Omega} I\left(1-H_{\varepsilon}(\Phi)\right) d x d y$, and $\int_{\Omega} \log (I)\left(1-H_{\varepsilon}(\Phi)\right) d x d y$ by three constant variables named $c_{e 1}, c_{e 2}$, and $c_{e 3}$; respectively, we can write:

$\left.\frac{\partial}{\partial t} S_{e}(\Phi+\psi t)\right|_{t=0}=\sigma S_{e}=\int_{\Omega} \delta_{\varepsilon}(\Phi)\left[\frac{-I c_{e 1}+c_{e 2}}{c_{e 1} c_{e 2}}+\frac{\log (I) c_{e 1}-c_{e 3}}{\left(c_{e 1}\right)^{2}}\right] \psi d x d y=\int_{\Omega} \delta_{\varepsilon}(\Phi)\left[C_{e}\right] \psi d x d y$ where $C_{e}$ is an auxiliary vector. Thus (B1) is expressed as:

$$
\lim _{t \rightarrow 0} \frac{1}{t}(F(\Phi+t \psi)-F(\Phi)) \approx \lim _{t \rightarrow 0} \frac{1}{t}(F(\Phi)+\sigma F t \psi-F(\Phi))=\sigma F \psi
$$


where

$F(\Phi)=\mu \int_{\Omega}|\nabla H(\Phi)| d x d y-\frac{A_{i}}{2 S_{i}}\left(\log \left(2 S_{i}\right)+1\right)-$

$A_{i} \log \left(\Gamma\left(\frac{1}{2 S_{i}}\right)\right)-\frac{A_{e}}{2 S_{e}}\left(\log \left(2 S_{e}\right)+1\right)-A_{e} \log \left(\Gamma\left(\frac{1}{2 S_{e}}\right)\right)$,

Or equivalently:

$$
\begin{aligned}
& F(\Phi)=\mu \int_{\Omega} \delta_{\varepsilon}(\Phi)|\nabla \Phi| d x d y-\left(\frac{\int_{\Omega} H_{\varepsilon}(\Phi) d x d y}{2 S_{i}}\right)\left(\log \left(2 S_{i}\right)+1\right)- \\
& \left(\int_{\Omega} H_{\varepsilon}(\Phi) d x d y\right) \log \left(\Gamma\left(\frac{1}{2 S_{i}}\right)\right)-\left(\frac{\int_{\Omega}\left(1-H_{\varepsilon}(\Phi)\right) d x d y}{2 S_{e}}\right)\left(\log \left(2 S_{e}\right)+1\right)- \\
& \left(\int_{\Omega}\left(1-H_{\varepsilon}(\Phi)\right) d x d y\right) \log \left(\Gamma\left(\frac{1}{2 S_{e}}\right)\right) . \\
& \lim _{t \rightarrow 0} \frac{1}{t}(F(\Phi+t \psi)-F(\Phi)) \approx \sigma F \psi=\int_{\Omega} \mu\left(\delta_{\varepsilon}^{\prime}(\Phi)|\nabla \Phi| \psi+\delta_{\varepsilon}(\Phi) \frac{\nabla \Phi \nabla \psi}{|\nabla \psi|}\right) d x d y- \\
& {\left[\left(\frac{\left(\int_{\Omega} \delta_{\varepsilon}(\Phi) d x d y\right)\left(2 S_{i}\right)-\left(2 \sigma S_{i}\right)\left(\int_{\Omega} H_{\varepsilon}(\Phi) d x d y\right)}{\left(2 S_{i}\right)^{2}}\right)\left(\log \left(2 S_{i}\right)+1\right)+\left(\frac{\int_{\Omega} H_{\varepsilon}(\Phi) d x d y}{2 S_{i}}\right)\left(\frac{\sigma S_{i}}{S_{i}}\right)+\right.} \\
& \left(\int_{\Omega} \delta_{\varepsilon}(\Phi) d x d y\right) \log \left(\Gamma\left(\frac{1}{2 S_{i}}\right)\right)-\left(\int_{\Omega} H_{\varepsilon}(\Phi) d x d y\right) \Psi\left(\frac{1}{2 S_{i}}\right)\left(\frac{\sigma S_{i}}{2 S_{i}^{2}}\right)+ \\
& \left(\frac{\left(-\int_{\Omega} \delta_{\varepsilon}(\Phi) d x d y\right)\left(2 S_{e}\right)-\left(2 \sigma S_{e}\right)\left(\int_{\Omega}\left(1-H_{\varepsilon}(\Phi)\right) d x d y\right)}{\left(2 S_{e}\right)^{2}}\right)\left(\log \left(2 S_{e}\right)+1\right)+\left(\frac{\int_{\Omega}\left(1-H_{\varepsilon}(\Phi)\right) d x d y}{2 S_{e}}\right)\left(\frac{\sigma S_{e}}{S_{e}}\right)- \\
& \left.\left(\int_{\Omega} \delta_{\varepsilon}(\Phi) d x d y\right) \log \left(\Gamma\left(\frac{1}{2 S_{e}}\right)\right)-\left(\int_{\Omega}\left(1-H_{\varepsilon}(\Phi)\right) d x d y\right) \Psi\left(\frac{1}{2 S_{e}}\right)\left(\frac{\sigma S_{e}}{2 S_{e}{ }^{2}}\right)\right] \psi,
\end{aligned}
$$

Substituting $\sigma S_{i}$ and $\sigma S_{e}$ from (B7) and (B9) into (B13) and using the linearity property of the integral, we have:

$$
\begin{aligned}
& \lim _{t \rightarrow 0} \frac{1}{t}(F(\Phi+t \psi)-F(\Phi))=\int_{\Omega} \mu\left(\delta_{\varepsilon}^{\prime}(\Phi)|\nabla \Phi| \psi+\delta_{\varepsilon}(\Phi) \frac{\nabla \Phi \nabla \psi}{|\nabla \psi|}\right) d x d y-\int_{\Omega} \delta_{\varepsilon}(\Phi)\left[\frac{\left(1+\log \left(2 S_{i}\right)\right)}{2 S_{i}}+\right. \\
& \left.\log \left(\Gamma\left(\frac{1}{2 S_{i}}\right)\right)-\frac{\left(1+\log \left(2 S_{e}\right)\right)}{2 S_{e}}-\log \left(\Gamma\left(\frac{1}{2 S_{e}}\right)\right)\right] \psi d x d y-
\end{aligned}
$$


$\int_{\Omega} \delta_{\varepsilon}(\Phi)\left[-\frac{C_{i}}{2 S_{i}^{2}} \log \left(2 S_{i}\right) c_{i 1}-\Psi\left(\frac{1}{2 S_{i}}\right)\left(\frac{C_{i}}{2 S_{i}^{2}}\right) c_{i 1}-\right.$

$\left.\frac{C_{e}}{2 S_{e}^{2}} \log \left(2 S_{e}\right) c_{e 1}-\Psi\left(\frac{1}{2 S_{e}}\right)\left(\frac{C_{e}}{2 S_{e}^{2}}\right) c_{e 1}\right] \psi^{2} d x d y=0$,

where $\Psi$ is the Digamma function and $\Gamma$ is the Gamma function. Using Green's theorem, we achieve:

$\int_{\Omega} \mu \delta_{\varepsilon}^{\prime}(\Phi)|\nabla \Phi| \psi d x d y+\int_{\partial \Omega} \mu \frac{\delta_{\varepsilon}(\Phi)}{|\nabla \Phi|} \frac{\partial \Phi}{\partial n} \psi d s-\int_{\Omega} \mu \nabla\left(\delta_{\varepsilon}(\Phi) \frac{\nabla \Phi}{|\nabla \Phi|}\right) \psi d x d y-\int_{\Omega} \delta_{\varepsilon}(\Phi)\left[\frac{\left(1+\log \left(2 S_{i}\right)\right)}{2 S_{i}}+\right.$

$\left.\log \left(\Gamma\left(\frac{1}{2 S_{i}}\right)\right)-\frac{\left(1+\log \left(2 S_{e}\right)\right)}{2 S_{e}}-\log \left(\Gamma\left(\frac{1}{2 S_{e}}\right)\right)\right] \psi d x d y-$

$\int_{\Omega} \delta_{\varepsilon}(\Phi)\left[-\frac{C_{i}}{2 S_{i}^{2}} \log \left(2 S_{i}\right) c_{i 1}-\Psi\left(\frac{1}{2 S_{i}}\right)\left(\frac{C_{i}}{2 S_{i}^{2}}\right) c_{i 1}-\right.$

$\left.\frac{C_{e}}{2 S_{e}^{2}} \log \left(2 S_{e}\right) c_{e 1}-\Psi\left(\frac{1}{2 S_{e}}\right)\left(\frac{C_{e}}{2 S_{e}^{2}}\right) c_{e 1}\right] \psi^{2} d x d y=0$,

By merging, we have:

$-\int_{\Omega} \mu \delta_{\varepsilon}(\Phi) \nabla\left(\frac{\nabla \Phi}{|\nabla \Phi|}\right) \psi d x d y+\int_{\partial \Omega} \mu \frac{\delta_{\varepsilon}(\Phi)}{|\nabla \Phi|} \frac{\partial \Phi}{\partial n} \psi d s-\int_{\Omega} \delta_{\varepsilon}(\Phi)\left[\frac{\left(1+\log \left(2 S_{i}\right)\right)}{2 S_{i}}+\log \left(\Gamma\left(\frac{1}{2 S_{i}}\right)\right)-\right.$

$\left.\frac{\left(1+\log \left(2 S_{e}\right)\right)}{2 S_{e}}-\log \left(\Gamma\left(\frac{1}{2 S_{e}}\right)\right)\right] \psi d x d y-\int_{\Omega} \delta_{\varepsilon}(\Phi)\left[-\frac{C_{i}}{2 S_{i}^{2}} \log \left(2 S_{i}\right) c_{i 1}-\right.$

$\left.\Psi\left(\frac{1}{2 S_{i}}\right)\left(\frac{C_{i}}{2 S_{i}^{2}}\right) c_{i 1}-\frac{C_{e}}{2 S_{e}^{2}} \log \left(2 S_{e}\right) c_{e 1}-\Psi\left(\frac{1}{2 S_{e}}\right)\left(\frac{C_{e}}{2 S_{e}{ }^{2}}\right) c_{e 1}\right] \psi^{2} d x d y=0$,

This expression must vanish for all test function $\psi$. We obtain:

$0=\delta_{\varepsilon}(\Phi)\left[\mu \operatorname{div}\left(\frac{\nabla \Phi}{|\nabla \Phi|}\right)+\frac{\left(1+\log \left(2 S_{i}\right)\right)}{2 S_{i}}-\frac{C_{i}}{2 S_{i}^{2}} \log \left(2 S_{i}\right) c_{i 1}+\log \left(\Gamma\left(\frac{1}{2 S_{i}}\right)\right)-\Psi\left(\frac{1}{2 S_{i}}\right)\left(\frac{C_{i}}{2 S_{i}^{2}}\right) c_{i 1}-\right.$

$\left.\frac{\left(1+\log \left(2 S_{e}\right)\right)}{2 S_{e}}-\frac{C_{e}}{2 S_{e}^{2}} \log \left(2 S_{e}\right) c_{e 1}-\log \left(\Gamma\left(\frac{1}{2 S_{e}}\right)\right)-\Psi\left(\frac{1}{2 S_{e}}\right)\left(\frac{C_{e}}{2 S_{e}^{2}}\right) c_{e 1}\right]$,

and also:

$$
\frac{\delta_{\varepsilon}(\Phi)}{|\nabla \Phi|} \frac{\partial \Phi}{\partial n}=0 \quad \text { on } \partial \Omega
$$


Note that with $\varepsilon$ approaching zero, $H_{\varepsilon}$ and $\delta_{\varepsilon}$ in equations of $c_{i 1}$ and $c_{e 1}$ converge to $H$ and $\delta$. So the associated flow, in agreement with (13), can be written as follows:

$$
\begin{aligned}
& \partial_{t} \Phi=\delta(\Phi)\left[\mu \operatorname{div}\left(\frac{\nabla \Phi}{|\nabla \Phi|}\right)+\frac{\left(1+\log \left(2 S_{i}\right)\right)}{2 S_{i}}-\frac{C_{i}}{2 S_{i}^{2}} \log \left(2 S_{i}\right) c_{i 1}+\log \left(\Gamma\left(\frac{1}{2 S_{i}}\right)\right)-\Psi\left(\frac{1}{2 S_{i}}\right)\left(\frac{C_{i}}{2 S_{i}{ }^{2}}\right) c_{i 1}-\right. \\
& \left.\frac{\left(1+\log \left(2 S_{e}\right)\right)}{2 S_{e}}-\frac{C_{e}}{2 S_{e}^{2}} \log \left(2 S_{e}\right) c_{e 1}-\log \left(\Gamma\left(\frac{1}{2 S_{e}}\right)\right)-\Psi\left(\frac{1}{2 S_{e}}\right)\left(\frac{C_{e}}{2 S_{e}{ }^{2}}\right) c_{e 1}\right] .
\end{aligned}
$$

\section{References}

American Cancer Society, 2006. American Cancer Society: Breast Cancer Facts \& Figures 20052006. pp. 1-28.

Andrews, S., McIntosh, C., Hamarneh, Gh., 2011. Convex Multi-Region Probabilistic Segmentation with Shape Prior in the Isometric Logratio Transformation Space. IEEE International Conference on Computer Vision (IEEE ICCV), pp. 2096-2103.

Ayed, I.B., Mitiche, A., Belhadj, Z., 2005. Multiregion Level-Set Partitioning of Synthetic Aperture Radar Images. PAMI, pp. 793-800.

Azzalini, A., 1996. Statistical Inference-Based on the Likelihood. New York: Chapman and Hall. Ball, J.E., Bruce, L.M., 2007a. Digital mammographic computer aided diagnosis (CAD) using adaptive level set segmentation. Proceedings of the 29th Annual International Conference of the IEEE EMBS Cité Internationale, Lyon, France.

Ball, J.E., Bruce, L.M., 2007b. Digital mammogram spiculated mass detection and spicule segmentation using level sets. Proceedings of the 29th Annual International Conference of the IEEE EMBS Cité Internationale, Lyon, France.

Catarious, D.M., Baydush, A.H., Floyd Jr., C.E., 2004. Incorporation of an iterative, linear segmentation routine into a mammographic mass CAD system. Medical Physics, vol. 31, no. 6, pp. 1512-1520. 
Chan, T.F., Vese, L.A., 2001. Active contours without edges. IEEE Trans. on Image Processing, vol. 10, no. 2, pp. 266-277.

Changizi, N., Hamarneh, Gh., 2010. Probabilistic Multi-Shape Representation using an Isometric Log-Ratio Mapping. Lecture Notes in Computer Science, Medical Image Computing and Computer-Assisted Intervention (MICCAI), pp. 563-570.

Chesnaud, C., Re' fre' gier, Ph., Boulet, V., 1999. Statistical Region Snake-Based Segmentation Adapted to Different Physical Noise Models. IEEE Transactions on Pattern Analysis and Machine Intelligence, vol. 21, pp. 1145-1157.

Choi, S. C., Wette, R., 1969. Maximum Likelihood Estimation of the Parameters of the Gamma Distribution and Their Bias. Technometrics, 11(4): 683-690.

Demirkazık, F.B., Gülsün, M., Firat, P., 2003. Mammographic feature of nonpalpable spiculated lesions. Clinical Imaging, vol. 27, pp. 293-297.

Egan, R.L., 1988. Breast Imaging: Diagnosis and Morphology of Breast Diseases. Philadelphia, PA: W. B. Saunders Co.

Freedman, D., Zhang, T, 2004. Active contours for tracking distributions. IEEE Trans. on Image Processing, vol. 13, no. 4, pp. 518-526.

Georgiou, G., Cohen, F. S., 1998. Statistical characterization of diffuse scattering in ultrasound images. IEEE Trans. Ultrason., Ferroelect., Freq. Contr., vol. 45, no. 1, pp. 57-64.

Georgiou, T., Michailovich, O., Rathi, Y., Malcolm., J., Tannenbaum A., 2007. Distribution metrics and image segmentation. Linear Algebra and its Applications, vol. 425, pp. 663-672.

Gilbert F., Astley, S., Gillan, M., Agbaje, OF, Wallis, MG, James. J, Boggis, CR, Duffy, SW, 2008. Single reading with computer-aided detection for screening mammography. New England Journal of Medicine, pp. 1675-1684. 
Guliato, D., Rangayyan, R. M., Carnielli, W. A., Zuffo, J. A., Desautels, J. E. L., 2003a. Segmentation of breast tumors in mammograms using fuzzy sets. Journal of Electronic Imaging, 12(3):369-378.

Guliato, D., Rangayyan, R. M., Carnielli, W. A., Zuffo, J. A., Desautels, J. E. L., 2003b. Fuzzy fusion operators to combine results of complementary medical image segmentation techniques. Journal of Electronic Imaging, 12(3):379-389.

Gupta, L., Srinath, M. D., 1987. Contour sequence moments for the classification of closed planar shapes. Pattern Recognition, 20(3):267-272.

Horritt, 1999. A statistical active contour model for SAR image segmentation. Image and Vision Computing, pp. 213-224.

Jay, A., Baker, MD, Eric, L., Rosen, MD, Michele, M., Crockett, MD, Joseph, Y., Lo, 2004. Accuracy of Segmentation of a Commercial Computer-aided Detection System for Mammography, Radiology journal.

Jaccard, P., 1908. Nouvelles recherches sur la distribution florale. Bulletin Société Vaudoise des Sciences Naturelles, vol. 44, pp. 223-270.

Jeske, J.M., Bernstein, J.R., Stull, M.A., 2000. Screening and Diagnostic Imaging. American Cancer Society Atlas of Clinical Oncology London, UK: B.C. Decker, pp. 41-63.

Kass, M., Witkin, A., Terzopoulos, D., 1998. Snakes: Active Contour Models. International journal of Computer Vision, vol. 1, pp. 321- 331.

Leventon, M.E., Grimson, W.E.L., Faugeras, O., 2000. Statistical shape influence in geodesic active contours, CVPR, pp. 316-323.

Martin, D., Refregier, P., Goudail, P., Guerault, F., 2004. Influence of the noise model on level set active contour segmentation. IEEE Transactions on Pattern Analysis and Machine Intelligence, Volume 26, Issue 6, $799-803$. 
Michailovich, O., Rathi, Y., Tannenbaum, A., 2007. Image Segmentation Using Active Contours Driven by the Bhattacharyya Gradient Flow. IEEE Trans. on Image Processing, vol. 16, pp. 2787 $-2801$.

Nascimento, J.C., Marques, J.S., 2005. Adaptive Snakes Using the EM Algorithm. IEEE Trans. Image Processing, pp. 1678-1686.

Osher, S., Paragios, N., 2003. Level Set Methods. in Geometric Level Set Methods in Imaging, Vision, and Graphics, Eds. New York, NY: Springer, pp. 1-20.

Paragios, N., 2000. Geodesic active regions and level set methods: contributions and applications in artificial vision. $\mathrm{PhD}$ in Computer Engineering. Sophia Antipolis, France: University of Nice.

Paragios, N., Deriche, R., 2000. Geodesic active contours and level sets for the detection and tracking of moving objects. IEEE Trans. Pattern Anal. Machine Intell., vol. 22, pp. 266-280.

Paragios, N., Deriche, R., 2002. Geodesic Active Regions: A new framework to deal with frame partition problems in Computer Vision. Journal of Visual Communication and Image Representation, vol. 13, pp. 249-268.

Pisano, E.D., Cole, E.B., Hemminger, B.M., Yaffe, M.J., Aylward, S.R., Maidment, A.D.A., Johnston, R.E., Williams, M.B., Niklason, L.T., Conant, E.F., Fajardo, L.L., Kopans, D.B., Brown, M.E., Pizer, S.M., 2000. Image Processing Algorithms for Digital Mammography: A Pictorial Essay. RadioGraphics, vol. 20, no. 5, pp. 1479-1491.

Rahmati, P., Ayatollahi, A., 2009. Maximum Likelihood Active Contours Specialized for Mammography Segmentation. The IEEE 2nd international conference on Biomedical Engineering and Informatics, Tianjin, China.

Rahmati, P., Hamarneh, G., Nussbaum, D., Adler, A., 2010. A New Preprocessing Filter for Digital Mammogram. International Conference on Image and Signal Processing 2010 (ICISP), LNCS 6134, Springer-Verlag Berlin Heidelberg, pp. 585-592, QUEBEC, Canada. 
Rangayyan, R. M., El-Faramawy, N. M., Desautels, J. E. L., Alim, O. A., 1997. Measures of acutance and shape for classification of breast tumors. IEEE Transactions on Medical Imaging, 16(6):799-810.

Rangayyan, R.M., 2005. Breast cancer and mammography. Biomedical Image Analysis. M.R. Neuman, Ed. Boca Raton, FL: CRC Press, pp. 22-27.

Rathi, Y., Michailovich, O., Malcolm, J., Tannenbaum, A., 2006. Seeing the unseen: segmenting with distributions. Proceedings of the eighth IASTED International Conference Signal and Image Processing, pp. 118-122, Honolulu, Hawaii, USA.

Refregier, Ph., Germain, O., Gaidon, T., 1997. Optimal snake segmentation of target and background with independent Gamma density probabilities, application to speckled and preprocessed images. Optical Communications, pp. 382-388.

Rousson, M., Paragios, N., 2002, Shape priors for level set representations, ECCV, pp. 78-92.

Sahiner, B., Chan, H.-P., Petrick, N., Helvie, M.A., Goodsitt, M.M., 1998. Computerized characterization of masses on mammograms: The rubber band straightening transform and texture analysis. Medical Physics, vol. 25, no. 4, pp. 516-526, Apr. 1998.

Sahiner, B., Petrick, N., Heang-Ping, C., Hadjiiski, L.M., Paramagul, C., Helvie, M.A., Gurcan, M.N., 2001. Computer-aided characterization of mammographic masses: accuracy of mass segmentation and its effects on characterization. IEEE Trans. on Medical Imaging, vol. 20, no. 12, pp. $1275-1284$.

Sandhu, R., Georgiou, T., Tannenbaum, A., 2008. A new distribution metric for image segmentation. Proc. SPIE, Vol. 6914, 691404.

Sarti, A., Corsi, C., Mazzini, E., Lamberti, C., 2005. Maximum Likelihood Segmentation of Ultrasound Images with Rayleigh Distribution. IEEE Trans. on Ultrasonics, Ferroelectrics, and Frequency Control, vol. 52, no. 6, pp 947-960. 
Sethian, J. A., 1996. Level Set Methods: Evolving Interface in Geometry, Fluid Mechanics, Computer Vision, and Materials Science. Cambridge Univ. Press.

Sethian, J.A., 2002. Level Set Methods and Fast Marching Methods: Evolving Interfaces in Computational Geometry, Fluid Mechanics, Computer Vision, and Materials Science, $2^{\text {nd }}$ ed. Cambridge, UK: Cambridge University Press, Chap. 10.

Shen, L., 1992. Shape analysis of mammographic calcifications. Master's thesis, Department of Electrical and Computer Engineering, University of Calgary, Calgary, Alberta, Canada.

Shen, L., Rangayyan, R. M., Desautels, J. E. L., 1993. Detection and classification of mammographic calcifications. International Journal of Pattern Recognition and Artificial Intelligence, 7(6):1403-1416.

Shen, L., Rangayyan, R. M., Desautels, J. E. L., 1994. Application of shape analysis to mammographic calcifications. IEEE Transactions of Medical Imaging, 13(2):263-274.

Suckling, J., Parker, J., Dance, D., Astley, S., Hutt, I., Boggis, C., Rickets, I., Stamatakis, E., Cerneaz, N., Kok, S., Taylor, P., Betal, D., Savage, J., 1994. The mammographic images analysis society digital mammogram database. Digital Mammography, pp. 375-378.

Sussman, M., Smereka, P., Osher, S., 1994. A level set approach for computing solutions to incompressible two-phase flow. J. Computat. Phys., vol. 119, pp. 146-159.

The Mosby Medical Encyclopedia, 1992. Revised ed. New York, NY: The Penguin Group. Yezzi, A., Kichenassamy, Jr., S., Kumar, A., Olver, P., Tannenbaum, A., 1997. A geometric snake model for segmentation of medical imagery. IEEE Trans. on Medical Imaging, vol. 16, no. 2, pp. 199-209. 